\title{
Superextensions of metrizable continua are Hilbert cubes
}

\author{
by
}

Jan van Mill * (Madison, Wisc.)

Abstract. We prove that the superextension $\lambda X$ of $X$ is a Hilbert cube if and only if $X$ is a nondegenerate metrizable continuum, thus proving a conjecture of de Groot.

0. Introduction. The aim of this paper is to prove the generalized de Groot conjecture (stated in Verbeek [27]) that the superextension $\lambda X$ of $X$ is a Hilbert cube if and only if $X$ is a nondegenerate metrizable continuum. In our previous paper [14] we proved that the superextension of the closed unit interval $\lambda I$ is a Hilbert cube. With a similar technique we show here that if $X$ is a finite topological sum of closed unit segments that the maximal connected superextension $\mu X$ of $X$ is a Hilbert cube. Then, by an approximation technique, this result is used to prove that superextensions of finite connected graphs are Hilbert cubes. By a similar approximation technique, using deep results of Curtis and Schori [9], [10], it then follows that $\lambda X$ is a Hilbert cube for every nondegenerate connected polyhedron. By a result of van Mill and Van de Vel [20] this fact suffices to prove the generalized de Groot conjecture.

Throughout this paper joint results of Marcel Van de Vel and the author concerning subbase convexity theory are used extensively. We would like to thank Marcel Van de Vel for his stimulating enthusiasm and for many helpful comments.

1. The spaces $\mu X$. Let $\mathscr{S}$ be a subbase for the closed subsets of a topological space. A subsystem $\mathscr{H} \subset \mathscr{S}$ is called a linked system provided that every two of its members meet. A maximal linked system, or, mls, is a linked system $\mathscr{A} \subset \mathscr{S}$ not properly contained in another linked system $\mathscr{N} \subset \mathscr{S}$. Let $\lambda(X, \mathscr{S})$ denote the collection of mls's in $\mathscr{S}$. For each $A \subset X$ define $A^{+} \subset \lambda(X, \mathscr{S})$ by

$$
A^{+}:=\{\mathscr{M} \in \lambda(X, \mathscr{S}) \mid \exists M \in \mathscr{M}: \mathscr{M} \subset A\} .
$$

We topologize $\lambda(X, \mathscr{S})$ by taking the collection $\mathscr{S}^{+}=\left\{S^{+} \mid S \in \mathscr{S}\right\}$ is a closed subbase. With this subbase $\lambda(X, \mathscr{S})$ is called the superextension of $X$ with respect to $\mathscr{S}$. The subbase $\mathscr{S}^{+}$has the property that every linked subcollection of it has

* Research supported by the Netherlands Organization for the Advancement of Pure Research (Z.W. O); Juliana van Stolberglaan 148, 's-Gravenhage, The Netherlands. 
nonempty intersection. Such a subbase is called binary. $\lambda(X, \mathscr{P})$ is Hausdorff whenever $\mathscr{S}$ is normal, i.e. disjoint subbase sets are separated by disjoint complements of subbase sets. It is easily seen that $\mathscr{S}$ is normal iff $\mathscr{S}^{+}$is normal. A subbase $\mathscr{S}$ for $X$ is called a $T_{1}$-subbase if for each $x \in X$ and $S \in \mathscr{S}$ with $x \notin S$ there is an $S_{0} \in \mathscr{S}$ such that $x \in S_{0}$ and $S_{0} \cap S=\varnothing$. If $\mathscr{S}$ is a $T_{1}$-subbase then the mapping $i: X \rightarrow \lambda(X, \mathscr{S})$ defined by $i(x):=\{S \in \mathscr{S} \mid x \in S\}$ is an embedding. We will always identify $X$ and $i[X]$. If $\mathscr{S}$ consists of all the closed subsets of $X$ then $\lambda(X, \mathscr{S})$ is denoted by $\lambda X$ and is called the superextension of $X$. In this paper we will prove that $\lambda X$ is homeomorphic to the Hilbert cube if and only if $X$ is a nondegenerate metrizable continuum, thus proving a conjecture of De Groot. For more information concerning superextensions, see Verbeek [27] and van Mill [16].'

For the remainder of this section let $X$ be a finite topological sum of nondegenerate continua. Let $2^{X}$ be the hyperspace of $X$. (Recall that for any topological space $X$ the hyperspace $2^{X}$ is the space with underlying set the set of all nonvoid closed subsets of $X$ topologized by taking the collection

$$
\left\{\langle F\rangle \mid F \in 2^{X}\right\} \cup\left\{\langle F, X\rangle \mid F \in 2^{X}\right\}
$$

as a closed subbase, where, for all $A_{1}, \ldots, A_{n} \subset X$ the set $\left\langle A_{1}, \ldots, A_{n}\right\rangle \subset 2^{X}$ is defined by

$$
\left\langle A_{1}, \ldots, A_{n}\right\rangle=\left\{F \in 2^{X} \mid F \subset \bigcup_{i \leqslant n} A_{i} \text { and } F \cap A_{i} \neq \varnothing \text { for all } i \leqslant n\right\} .
$$

It is well known that $2^{X}$ is compact iff $X$ is compact (cf. Michael [13]). This fact will be used without explicit reference in' the remaining part of this paper. For many strong results concerning hyperspaces, see [7], [8], [9], [10], [22], [23], [24] and [28]). Define

$$
\mathscr{T}(X):=\left\{A \in 2^{X} \mid A \text { is not open }\right\} \cup\{\varnothing, X\} .
$$

1.1. LemMA. $\mathscr{T}(X)$ is a normal closed $T_{1}$-subbase for $X$.

Proof. That $\mathscr{T}(X)$ is a closed subbase is trivial. Also, since $X$ has no isolated points, $\mathscr{T}(X)$ is a $T_{1}$-subbase. Hence it suffices to prove that $\mathscr{T}(X)$ is normal. To this end, take $T_{0}, T_{1} \in \mathscr{T}(X)$ such that $T_{0} \cap T_{1}=\varnothing$. It is easy to construct a surjective Urysohn mapping $f: X \rightarrow I$ such that $f\left[T_{i}\right]=i(i \in\{0,1\})$. Then, since $f$ is surjective, $\left\{f^{-1}\left[0, \frac{1}{2}\right], f^{-1}\left[\frac{1}{2}, 1\right]\right\} \subset \mathscr{T}(X)$; consequently $\left\{f^{-1}\left[0, \frac{1}{2}\right], f^{-1}\left[\frac{1}{2}, 1\right]\right\}$ is the desired covering of $X$ by elements of $\mathscr{T}(X)$.

For simplicity of notation write $\mu X:=\lambda(X, \mathscr{T}(X))$. Notice that $\mu X=\lambda X$ in case $X$ is connected.

We need the following result (cf. van Mill [16], Theorem 2.5.1).

1.2. THEOREM. Let $X$ be a topological space and let $\mathscr{S}$ be a normal closed $T_{1}$-subbase for $X$. Then the following properties are equivalent:

(i) $\lambda(X, \mathscr{S})$ is connected;

(ii) $\lambda(X, \mathscr{S})$ is connected and locally connected;

(iii) for all nonvoid $S_{0}, S_{1} \in \mathscr{S}:\left(S_{0} \cap S_{1}=\varnothing \Rightarrow S_{0} \cup S_{1} \neq X\right)$.
1.3. COROLlaRY. Let $X$ be a finite sum of nondegenerate metrizable continua. Then $\mu X$ is an AR.

Proof. $\mathscr{T}(X)$ clearly satisfies condition (iii) of Theorem 1.2. Consequently $\lambda(X, \mathscr{T}(X))$ is connected. In addition $\lambda(X, \mathscr{T}(X))$ is metrizable by a result of Verbeek [27]. Hence $\lambda(X, \mathscr{T}(X))$ is a connected metrizable space with a binary normal subbase (cf. Lemma 1.1). However such a space is an AR by a result of van Mill [14].

We think of $\mu X$ as the maximal connected superextension of $X$. It can be shown that for every connected superextension $\lambda(X, \mathscr{S})$ of $X$ with respect to a normal $T_{1}$-closed subbase $\mathscr{S}$ there is a continuous surjection $f: \mu X \rightarrow \lambda(X, \mathscr{S})$ which extends the identity on $X$ and which in addition respects the canonical convexity structures of $\mu X$ and $\lambda(X, \mathscr{S})$ (cf. van Mill and Van de Vel [19]).

In Section 6 we will show that $\mu X$ is a Hilbert cube if $X$ is a finite sum of nondegenerate metrizable continua.

2. Convexity preserving mappings. Let $X$ be a space which possesses a binary normal closed subbase $\mathscr{S}$. A nonempty closed subset $C \subset X$ is called $\mathscr{S}$-closed provided there is a subfamily $\mathscr{C} \subset \mathscr{S}$ such that $C=\bigcap \mathscr{C}$. Let $H(X, \mathscr{S})$ be the set of all $\mathscr{S}$-closed subsets of $X$. We.topologize $H(X, \mathscr{S})$ by regarding it to be a subspace of the hyperspace $2^{X}$ of $X$. For each $A \subset X$ define $I_{\mathscr{S}}(A) \in H(X, \mathscr{S})$ by

$$
I_{\mathscr{H}}(A):=\bigcap\{S \in \mathscr{S} \mid A \subset S\} .
$$

The set $I_{\mathscr{H}}(A)$ is called the $\mathscr{S}$-closure of $A$ and $I_{\mathscr{S}}$ is called the convex closure operator. If $A$ is a two point set, say $A=\{x, y\}$, then we usually write $I_{y}(x, y)$ instead of $I_{\mathscr{S}}(\{x, y\})$. Let $L(X, \mathscr{S})$ denote the subspace of the hyperspace $2^{H(X, \mathscr{S})}$, consisting of all nonempty, closed (in $H(X, \mathscr{S})$ ), and linked systems $\mathscr{L} \subset H(X, \mathscr{S})$. We need the following results (cf. van Mill and Van de Vel [17]).

2.1. THEOREM. Let $X$ be a topological space which possesses a binary normal closed subbase $\mathscr{S}$. Then

(i) The convex closure operator $I_{\mathscr{S}}: 2^{X} \rightarrow H(X, \mathscr{S})$ is continuous;

(ii) the mapping $p: X \times H(X, \mathscr{S}) \rightarrow X$ defined by

$$
\{p(x, A)\}=\bigcap_{a \in A} I_{\mathscr{P}}(x, a) \cap A
$$

is continuous ( $p$ is called the nearest-point mapping of $X$ );

(iii) a closed set $A \subset X$ is $\mathscr{S}$-closed iff $\forall x, y \in A: I_{\mathscr{S}}(x, y) \subset A$; .

(iv) the intersection operator $\cap: L(X, \mathscr{S}) \rightarrow H(X, \mathscr{S})$ which sends each $\mathscr{L} \in L(X, \mathscr{P})$ onto $\cap \mathscr{L}$, is continuous.

Notice that for each $A \in H(X, \mathscr{S})$ the restriction of $p$ to $X \times\{A\}$ is a retraction of $X$ onto $A$. This is a very useful result and already had a variety of applications (cf. Van de Vel [26], van Mill and Van de Vel [18], van Mill [15], Szymański [25]). 
The convex closure operator of $\mu X$ (resp. $\lambda X$ ) with respect to its canonical binary normal subbase $\mathscr{T}(X)^{+}=\left\{T^{+} \mid T \in \mathscr{T}(X)\right\}$ (resp. $\left(2^{X}\right)^{+}=\left\{F^{+} \mid F \in 2^{X}\right\}$ ) will simply be denoted by $I$.

Let $X$ and $Y$ bo spaces, and let $\mathscr{S}$ and $\mathscr{T}$ be binary normal subbases for, respectively, $X$ and $Y$. A function $f: X \rightarrow Y$ is called a convexity preserving map (briefly: a cp map) relative to $\mathscr{S}$ and $\mathscr{T}$ if for each $T \in H(Y, \mathscr{T})$ it is true that $f^{-1}(T) \in H(X, \mathscr{S}) \cup\{\varnothing\}$ (cf. van Mill and Van de Vel [19]). In this case we shall write

$$
f:(X, \mathscr{S}) \rightarrow(Y, \mathscr{T}) .
$$

Notice that a cp mapping is automatically continuous.

If $X$ is a space, then it is easily seen that each $\mathscr{M} \in \lambda X$ regarded as subspace of $2^{X}$ is closed in $2^{X}$. This suggests a mapping from $\lambda X$ inte $2^{2^{X}}$ which sends each $\mathscr{M} \in \lambda X$ onto $\mathscr{M} \in 2^{2^{x}}$. This mapping is obviously one to one and it is quite surprising that for compact Hausdorff $X$ it is also continuous (cf. van Mill and Van de Vel [20]). Hence for compact Hausdorff $X$ we may regard $\lambda X$ to be a subspace of $2^{2^{x}}$. Often it is useful to do so (cf. Section 6 and the following theorem).

2.2. Theorem. Let $X$ and $Y$ be compact Hausdorff spaces and let $F: X \rightarrow 2^{Y}$ be continuous. Fix $y \in Y$. Then the function $f: \lambda X \rightarrow \lambda Y$ defined by

$$
f(\mathscr{M}):=p\left(y, \bigcap_{M \in \mathscr{M}}(\bigcup F(M))^{+}\right)
$$

is a cp mapping.

Proof. Let $k(\lambda Y)$ be the space $H\left(\lambda Y,\left(2^{Y}\right)^{+}\right)$. Define a mapping $F^{*}: 2^{X} \rightarrow 2^{Y}$ by $F^{*}(A):=\bigcup F[A]$. It is easily seen that this mapping is continuous. This mapping extends to a continuous mapping $H: 2^{2^{x}} \rightarrow 2^{2^{x}}$ defined by $H(\mathscr{A}):=F^{*}[\mathscr{A}]$. Take $\mathscr{M} \in \lambda X \subset 2^{2^{x}}$. We claim that $H(\mathscr{M})$ is a linked system. Indeed, since $H(\mathscr{M})$ $=\{\bigcup F[M] \mid M \in \mathscr{M}\}$, this is a simple consequence of the linkedness of $\mathscr{M}$. Define a mapping $\varphi: 2^{Y} \rightarrow k(\lambda Y)$ by $\varphi(A):=A^{+}$. It is easily seen that this mapping is an embedding. This mapping extends to a mapping $\varphi^{*}: 2^{2^{X}} \rightarrow 2^{k(\lambda Y)}$ defined in the obvious way. We now have the following mappings

$$
\lambda X \stackrel{i}{\rightarrow} 2^{2^{x}} \stackrel{H}{\rightarrow} 2^{2^{Y}} \stackrel{\varphi^{*}}{\rightarrow} 2^{k(\lambda Y)}
$$

where $i$ is the inclusion. Let $\psi:=\varphi^{*} \circ H \circ i$. Then $\psi[\lambda X] \subset L\left(\lambda Y,\left(2^{Y}\right)^{+}\right)$as was pointed out above (notice that $A \cap B \neq \varnothing$ implies that $\varphi(A) \cap \varphi(B) \neq \varnothing$ ). Regard $\psi$ to be a mapping from $\lambda X$ to $L\left(\lambda Y,\left(2^{Y}\right)^{+}\right)$. Then consider the composition

$$
\lambda X \stackrel{\psi}{\longrightarrow} L\left(\lambda Y,\left(2^{Y}\right)^{+}\right) \stackrel{n}{\longrightarrow} k(\lambda Y) \stackrel{p(y,-)}{\longrightarrow} \lambda Y
$$

Since the intersection operator is continuous, cf. Theorem 2.1(iv), we conclude that $f$ being the composition of $\psi, \cap$ and $p(y,-)$, is continuous (cf. Theorem 2.1(ii)).

Let us prove now that $f$ is $\mathrm{cp}$. Indeed, take $T \in 2^{Y}$ and assume that there are $\mathscr{M}, \mathscr{N} \in f^{-1}\left(T^{+}\right)$and $\mathscr{P} \in I(\mathscr{M}, \mathscr{N})$ such that $\mathscr{P} \in I(\mathscr{M}, \mathscr{N})-f^{-1}\left(T^{+}\right)$. We will derive a contradiction; by the binarity of the subbase $\left(2^{Y}\right)^{+}$there are two cases:
Case 1. There is $P \in \mathscr{P}$ such that $(\cup F(P))^{+} \cap T^{+}=\varnothing$.

Suppose that $P \notin \mathscr{M}$ and that $P \notin \mathscr{N}$. Take $M \in \mathscr{M}$ and $N \in \mathscr{N}$ such that $P \cap M=\varnothing=N \cap P$. Then $P \cap(M \cup N)=\varnothing$ and since $\mathscr{P} \in I(M \cup N) \subset(M \cup N)^{+}$ this is a contradiction. Hence we may assume that, without loss of generality, $P \in \mathscr{A l}$. Then $f(\mathscr{H}) \in(\bigcup F(P))^{+} \cap T^{+}$, which is impossible.

Case 2. There is an $S \in 2^{Y}$ such that

(i) $y \in S$ and $S \cap \cup F(P) \neq \varnothing$ for all $P \in \mathscr{P}$;

(ii) $S \cap T=\varnothing$.

Since $f(\mathscr{M}) \in T^{+}$and since $S^{+} \cap T^{+}=\varnothing$ we can consider two cases:

(a) $\exists M \in \mathscr{M}: \cup F(M) \cap S=\varnothing$;

(b) $\exists S_{0} \in 2^{Y}$ such that $S_{0} \cap \bigcup F(M) \neq \varnothing$ for all $M \in \mathscr{A}$ and $y \in S_{0}$ and moreover $S_{0} \cap S=\varnothing$.

However case (b) cannot occur since $y \in S_{0} \cap S_{1}$. Hence we may assume that there is an $M \in \mathscr{M}$ such that $\bigcup F(M) \cap S=\varnothing$. In the same way there is an $N \in \mathscr{N}$ such that $\bigcup F(N) \cap S=\varnothing$. Then $F(M) \cup F(N) \subset\langle\cup F(M) \cup \cup F(N)\rangle$ and consequently $M \cup N \subset F^{-1}\langle\bigcup F(M) \cup \cup F(N)\rangle$. Since $I(\mathscr{M}, \mathscr{N}) \subset(M \cup N)^{+}$we conclude that $M \cup N \in \mathscr{P}$. Hence

$$
f(\mathscr{P}) \in(\bigcup\langle\bigcup F(M) \cup \cup F(N)\rangle)^{+}=(\bigcup F(M) \cup \cup F(N))^{+}
$$

and since $S \in f(\mathscr{P})$ this contradicts the linkedness of $f(\mathscr{P})$.

By the continuity of $f$ the set $f^{-1}\left(T^{+}\right)$is closed in $\lambda X$. By the characterization of Theorém 2.1(iii) we conclude that $I\left(f^{-1}\left(T^{+}\right)\right)=f^{-1}\left(T^{+}\right)$for all $T \in 2^{Y}$. This obviously implies that $f$ is a cp mapping.

The proof of the above theorem is unexpectedly difficult. There are other theorems concerning $\mathrm{cp}$ mappings which have a more straightforward proof. For later use let us mention one (cf. van Mill and Van de Vel [19]).

2.3. THEOREM. Let $\mathscr{S}$ and $\mathscr{T}$ be normal $T_{1}$-subbases for the spaces $X$ and $Y$, respectively, and let $f: X \rightarrow Y$ be a mapping such that $f^{-1}(T) \in \mathscr{S}$ for each $T \in \mathscr{T}$. Then there is a canonical cp mapping $\lambda(f): \lambda(X, \mathscr{S}) \rightarrow \lambda(Y, \mathscr{T})$ extending $f$. Moreover $\lambda(f)$ is the unique cp mapping which extends $f$.

That in the above circumstances there is a continuous mapping $\bar{f}: \lambda(X, \mathscr{S})$ $\rightarrow \lambda(Y, \mathscr{T})$ was first proved by G. A. Jensen (see Verbeek [27]). The mapping $\bar{f}$ can be described as follows

$$
\{f(\mathscr{M})\}=\cap\left\{T^{+} \mid T \in \mathscr{T} \text { and } f^{-1}(T) \in \mathscr{M}\right\} .
$$

The mapping $\lambda(f)$ of Theorem 2.3 is simply Jensen's $f$. Hence the only new fact is that $f$ is a cp mapping and that it is unique as cp mapping. This is of interest since simple examples show that there may exist more than one continuous extension. The mappings $\lambda(f)$ will be called Jensen mappings.

In [14] a normal $T_{1}$-subbase $\mathscr{S}$ for a topological space $X$ was called supernormal provided that for all $S \in \mathscr{S}$ and $A \in 2^{X}$ with $S \cap A=\varnothing$ there is an $S_{0} \in \mathscr{S}$ such that 
$A \subset S_{0}$ and $S \cap S_{0}=\varnothing$. If $\mathscr{T}$ is a normal $T_{1}$-subbase for $X$ and if $\mathscr{S}$ is a supernormal subbase for $X$ such that $\mathscr{S} \subset \mathscr{T}$ then the Jensen extension

$$
\lambda(\text { id) }: \lambda(X, \mathscr{T}) \rightarrow \lambda(X, \mathscr{S}),
$$

which extends the identity on $X$, can be described very easily. In [14] it was shown that for each $\mathscr{M} \in \lambda(X, \mathscr{T})$ the set $\mathscr{M} \cap \mathscr{S}$ is an mls in $\mathscr{S}$ and hence determines a point of $\lambda(X, \mathscr{S})$. It is straightforward to prove that $\lambda(\mathrm{id})(\mathscr{M})=\mathscr{M} \cap \mathscr{S}$ for all $\mathscr{M} \in \lambda(X, \mathscr{T})$. This observation will be used several times in the sequel. In addition, notice that $\lambda$ (id) is onto.

Also notice that if $X$ is a sum of finitely many nondegenerate continua the subbase $\mathscr{T}(X)$ for $X$ defined in Section 1 is supernormal.

Finally we mention another useful fact concerning cp mappings (cf. van Mill and Van de Vel [19]).

2.4. TheOREM. Let $f:(X, \mathscr{S}) \rightarrow(Y, \mathscr{T})$ be a $\mathrm{cp}$ surjection. Then for all $A \in H(X, \mathscr{S})$ we have that $f[A] \in H(Y, \mathscr{T})$.

The proof of this theorem heavily relies on the fact that $\mathscr{S}$ and $\mathscr{T}$ both are binary normal subbases.

2.5. Corollary. Let $f:(X, \mathscr{S}) \rightarrow(Y, \mathscr{T})$ be a cp surjection. Take $x \in X$ and $A \in H(X, \mathscr{S})$. Then $f(p(x, A))=p(f(x), f[A])$.

Proof. Since $p(x, A) \in A$ we have that $f(p(x, A)) \in f[A]$. Suppose now that $f(p(x, A)) \neq p(f(x), f[A])$. Then $I_{\mathscr{T}}(f(x), p(f(x), f[A]))$ does not contain $f(p(x, A))$. Therefore $f^{-1} I_{\mathscr{T}}(f(x), p(f(x), f[A]))$ does not contain $p(x, A)$. This is a contradiction, however, since $x \in f^{-1} I_{\mathscr{T}}(f(x), p(f(x), f[A])) \in H(X, \mathscr{S})$ and clearly $f^{-1} I_{\mathscr{T}}(f(x), p(f(x), f[A])) \cap A \neq \varnothing$.

2.6. Corollary. Let $f:(X, \mathscr{P}) \rightarrow(Y, \mathscr{T})$ be a cp surjection. Then for all $A \subset X$ we have that $f\left[I_{\mathscr{S}}(A)\right]=I_{\mathscr{T}}(f[A])$.

Proof. Since $f[A] \subset f\left[I_{\mathscr{S}}(A)\right]$, by Theorem 2.4 we conclude that

$$
I_{\mathscr{T}}(f[A]) \subset f\left[I_{\mathscr{S}}(A)\right] .
$$

On the other hand, $A \subset f^{-1} I_{\mathscr{T}}(f[A])$ and consequently, since $f$ is a cp mapping, $f\left[I_{\mathscr{S}}(A)\right] \subset I_{\mathscr{T}}(f[A])$. This proves equality.

3. Some remarks concerning $\lambda X$ and $\mu X$. This section contains some remarks which are useful in the sequel.

In [27] Verbeek proved that if $(X, d)$ is a compact metric space then there is a canonical metric $\bar{d}$ for $\lambda X$ such that $i:(X, d) \hookrightarrow(\lambda X, \bar{d})$ is an isometry (here $i$ is the canonical embedding of $X$ in $\lambda X$ sending $x \in X$ onto the $\operatorname{mls}\left\{A \in 2^{X} \mid x \in A\right\}$ ). This is a very useful result. Before describing Verbeek's [27] metric explicitly, let us first give some definitions.

If $(X, d)$ is a metric space then for all $A \subset X$ and $\varepsilon \geqslant 0$ define

$$
\begin{aligned}
& B_{\varepsilon}(A):=\{x \in X \mid d(x, A) \leqslant \varepsilon\}, \\
& U_{\varepsilon}(A):=\{x \in X \mid d(x, A)<\varepsilon\} .
\end{aligned}
$$

For any $A, B \in 2^{X}$ the Hausdorff distance $d_{H}(A, B)$ is defined by

$$
d_{H}(A, B):=\inf \left\{\varepsilon \geqslant 0 \mid A \subset U_{\varepsilon}(B) \text { and } B \subset U_{\varepsilon}(A)\right\} \text {. }
$$

In case $X$ is compact, $d_{H}$ is a metric for $2^{X}$.

Now assume that $(X, d)$ is compact. Verbeek [27] has given the following expressions for $\bar{d}$;

(1) $\quad \bar{d}(\mathscr{M}, \mathscr{N})=\sup _{M \in \mathscr{M}} \min _{N \in \mathscr{N}} d_{H}(M, N)$

(2) $\quad=\min \left\{\varepsilon \geqslant 0 \mid \forall M \in \mathscr{M}: B_{\varepsilon}(M) \in \mathscr{N}\right.$ and $\left.\forall N \in \mathscr{N}: B_{\varepsilon}(N) \in \mathscr{M}\right\}$

(3) $\quad=\min \left\{\varepsilon \geqslant 0 \mid \forall M \in \mathscr{M}: B_{\varepsilon}(M) \in \mathscr{N}\right\}$

(4) $\quad=\min \left\{\varepsilon \geqslant 0 \mid \forall N \in \mathscr{N}: B_{\varepsilon}(N) \in \mathscr{M}\right\}$.

In practical calculations the expressions (3) and (4) are the most useful.

Another very useful result is the following (cf. Verbeek [27]): $\lambda X$ has finitely many components if and only if $X$ has finitely many components. This is proved in the following way: let $C_{1}, \ldots, C_{n}$ be the collection of components of a space $X$. Let

$$
\pi: X \rightarrow\{1,2, \ldots, n\}
$$

be the decomposition. Let

$$
\lambda(\pi): \lambda X \rightarrow \lambda\{1,2, \ldots, n\}
$$

be the Jensen extension of $f$ (cf. Section 2) defined by

$$
\lambda(\pi)(\mathscr{M})=\left\{A \subset\{1,2, \ldots, n\} \mid \pi^{-1}(A) \in \mathscr{M}\right\} .
$$

Verbeek [27] proved that the components of $\lambda X$ coincide with the collection

$$
\left\{\lambda(\pi)^{-1}(\mathscr{M}) \mid \mathscr{M} \in \lambda\{1,2, \ldots, n\}\right\} .
$$

Since the space $\lambda\{1,2, \ldots, n\}$ is finite so is the number of components of $\lambda X$.

This has an important corollary. Let $f=\lambda($ id) $: \lambda X \rightarrow \mu X$ be the Jensen mapping (cf. Section 2). Then the function $\varphi: \lambda X \rightarrow \mu X \times \lambda\{1,2, \ldots, n\}$ defined by $\varphi(\mathscr{H}):=\langle f(\mathscr{H}), \lambda(\pi)(\mathscr{M})\rangle$ is an embedding. Indeed, it is obvious that $\varphi$ is continuous and hence it suffices to prove that $\varphi$ is one to one. Take distinct $\mathscr{M}, \mathscr{N} \in \lambda X$. There are $M \in \mathscr{M}$ and $N \in \mathscr{N}$ such that $M \cap N=\varnothing$. If $M \in \mathscr{T}(X)$ then there is an element of $\mathscr{T}(X)$ disjoint from $M$ and containing $N$. Hence we may assume that also $N \in \mathscr{T}(X)$. Then $f\left[N^{+}\right] \cap f\left[M^{+}\right]=\varnothing$ since $\mathscr{T}(X)$ is supernormal and consequently $f(\mathscr{M}) \neq f(\mathscr{N})$. If $M \notin \mathscr{T}(X)$ then $M$ is clopen and so $N$ can be taken to be the complement of $M$. Then clearly $\lambda(\pi)(\mathscr{M}) \neq \lambda(\pi)(\mathscr{N})$. Hence $\varphi$ is one to one.

We finish this section with a simple but useful result.

3.1. LEMMA. Let $X$ be a sum of finitely many nondegenerate metrizable continua. Let $F=\left\{x_{1}, \ldots, x_{n}\right\}\left(i<j \leqslant n \Rightarrow x_{i} \neq x_{j}\right)$ consisting of at least 3 points. Take a point 
$\mathscr{M} \in \mu X$ such that $\mathscr{H} \in\left(\bigcap_{j=1}^{n}\left(F-\left\{x_{j}\right\}\right)^{+}\right)-F$. Then for every $i \leqslant n$ there is an arc $J_{i} \subset F^{+}$ connecting $\mathscr{M}$ and $x_{i}$ while in addition $i<j \leqslant n$ implies that $J_{i} \cap J_{j}=\{\mathscr{M}\}$.

Proof. By Corollary $1.3 \mu X$ is an AR and hence a Peano continuum. Let $d$ be a convex metric for $\mu X$ (i.e. a metric for which $B_{\delta_{0}}\left(B_{\delta_{1}}(A)\right)=B_{\delta_{0}+\delta_{1}}(A)$ for every $A \in 2^{\mu X}$; there is a convex metric on every Peano continuum (cf. Bing [0]). It is easy to show that the function $F: 2^{\mu X} \times[0, \infty) \rightarrow 2^{\mu X}$ defined by $F(\langle A, t\rangle):=B_{t}(A)$ is continuous ( $F$ is sometimes called an expansion homotopy, cf. Curtis and Schori [8]). Without loss of generality we may assume that $d(\mu X \times \mu X)=[0,1]$. It now is easy to show that the collection

$$
\left\{B_{t}(\mathscr{M}) \mid t \in[0,1]\right\} \subset 2^{\mu X}
$$

regarded to be a subspace of $2^{\mu X}$ is homeomorphic to $[0,1]$. This implies that the collection

$$
\left\{I\left(B_{t}(\mathscr{M})\right) \mid t \in[0,1]\right\} \subset k(\mu X)
$$

$(k(\mu X)=\{A \subset \mu X \mid A \neq \varnothing$ and $I(A)=A\})$ is also homeomorphic to $[0,1]$ since $I$ preserves inclusions (recall that $I$ is continuous, see Theorem 2.1(i)). Let

$$
p: \mu X \times k(\mu X) \rightarrow \mu X
$$

be the nearest point mapping of $\mu X$. (cf. Theorem 2.1(ii)). For each $i \leqslant n$ define $H_{i} \subset \mu X$ by

$$
H_{i}:=\left\{p\left(x_{i}, I\left(B_{t}(\mathscr{M})\right)\right) \mid t \in[0,1]\right\} .
$$

It is clear that $H_{i}$ is a Peano continuum being a continuous image of $[0,1]$. In addition it contains both $x_{i}$ and $\mathscr{M}$ since

and

$$
p\left(x_{i}, I\left(B_{0}(\mathscr{M})\right)\right)=p\left(x_{i},\{\mathscr{M}\}\right)=\mathscr{M}
$$

$$
p\left(x_{i}, I\left(B_{1}(\mathscr{M})\right)\right)=p\left(x_{i}, I(\mu X)\right)=p\left(x_{i}, \mu X\right)=x_{i} .
$$

In addition, since $\left\{x_{i}, \mathscr{M}\right\} \subset F^{+}$we see that $I\left(x_{i}, \mathscr{M}\right) \subset F^{+}$and consequently $H_{i} \subset I\left(x_{i}, \mathscr{M}\right) \subset F^{+}$. Now take $i<j \leqslant n$. We claim that $H_{i} \cap H_{j}=\{\mathscr{M}\}$. To the contrary, assume there exist $s, t \in[0,1]$ such that

$$
p\left(x_{i}, I\left(B_{s}(\mathscr{M})\right)\right)=p\left(x_{j}, I\left(B_{t}(\mathscr{M})\right)\right) .
$$

Since $H_{i} \subset I\left(x_{i}, \mathscr{M}\right)$ and $x_{j} \notin I\left(x_{i}, \mathscr{M}\right)$ (this is easily seen) we conclude that $x_{j} \neq p\left(x_{j}, I\left(B_{t}(\mathscr{M})\right)\right)$ and consequently $x_{j} \notin I\left(B_{t}(\mathscr{M})\right)$. In the same way we find that $x_{i} \notin I\left(B_{s}(\mathscr{M})\right)$. It is clear that not both $s$ and $t$ are 0 . Assume that $s=0$. Then $t \neq 0$ and

$$
\mathscr{M}=p\left(x_{j}, I\left(B_{t}(\mathscr{M})\right)\right)
$$

This is disproved by the following
FACT. Suppose that $x \notin C \in k(\mu X)$. Then $p(x, C) \notin \operatorname{int}(C)$.

Indeed, let $q:=p(x, C)$. By the definition of $p$ it is easily seen that

$$
I(x, q) \cap C=\{q\}
$$

(in fact this property characterizes $q$ ). Since $I(x, q)$ is a retract of the connected space $\mu X$ (cf. Theorem 2.1(ii)) it is connected too. So if $q \in \operatorname{int}(C)$ then $I(x, q) \cap(C-\{q\}) \neq \varnothing$ which is impossible.

We conclude that $\{s, t\} \subset(0,1)$. Since $\mathscr{M} \in\left(F-\left\{x_{i}\right\}\right)^{+}$and since $\left(F-\left\{x_{i}\right\}\right)^{+}$ is nowhere dense in $\mu X$ (this is easily seen since $F-\left\{x_{i}\right\}$ is nowhere dense) there is a point $\mathscr{L} \in B_{s}(\mathscr{M})-\left(F-\left\{x_{i}\right\}\right)^{+}$. Then $x_{i}$ and $\mathscr{L}$ are both not elements of $\left(F-\left\{x_{i}\right\}\right)^{+}$; consequently

$$
I\left(x_{i}, \mathscr{L}\right) \cap\left(F-\left\{x_{i}\right\}\right)^{+}=\varnothing .
$$

Since $\mathscr{L} \in B_{s}(\mathscr{M}) \subset I B_{s}(\mathscr{M})$ we see that

$$
p\left(x_{i}, I\left(B_{s}(\mathscr{M})\right)\right) \in I\left(x_{i}, \mathscr{L}\right) .
$$

But $\left\{x_{j}, \mathscr{M}\right\} \subset\left(F-\left\{x_{i}\right\}\right)^{+}$which implies that

$$
p\left(x_{j}, I\left(B_{t}(\mathscr{M})\right)\right) \subset I\left(x_{j}, \mathscr{M}\right) \subset\left(F-\left\{x_{i}\right\}\right)^{+} .
$$

We conclude that $p\left(x_{i}, I\left(B_{s}(\mathscr{M})\right)\right) \neq p\left(x_{j}, I\left(B_{t}(\mathscr{M})\right)\right)$; contradiction. Now for each $i \leqslant n$ let $J_{i} \subset H_{i}$ be an arc connecting $x_{i}$ and $\mathscr{M}_{i}$. Then $\left\{J_{1}, \ldots, J_{n}\right\}$ is as desired.

Remark. It can be shown that the spaces $H_{i}(1 \leqslant i \leqslant n)$ defined in the proof of the previous lemma are itself all homeomorphic to $[0,1]$. Hence there is in fact no need for taking subspaces $J_{i} \subset H_{i}$.

4. Superextensions of finite sums of $[0,1]$. In [14] we showed that the superextension of the closed unit segment $I=[0,1]$ is homeomorphic to the Hilbert cube $Q$ by showing that it is the inverse limit of a sequence of Hilbert cubes with cellular bonding maps (a continuous surjection $f: Q \rightarrow Q$ is said to be cellular if each point inverse has trivial shape, that is, each point inverse is contractible in each neighborhood of itself; for shape theory we refer to Borsuk [1]). With the same construction we prove here that $\mu X$ is a Hilbert cube provided that $X$ is a topological sum of finitely many copies of $I$.

Let $X:=[0,1] \cup[2,3] \cup \ldots \cup[m-1, m]$. Define $E:=\left\{-2 \cdot 3^{k} \mid k=0,1,2, \ldots\right\}$ and for each $n \in E$ let $X$ be embedded in $I \times[0, m]$, preserving arc-length, as indicated in Figure 1.

All angles are $\frac{1}{2} \pi$ except the one at $\left\langle\frac{1}{2}, 0\right\rangle$ which is $\frac{1}{4} \pi$. Let

$\mathscr{S}=\left\{A \subset[0,1] \times[0, m] \mid\left(A=\pi_{0}^{-1}[0, x] \vee A=\pi_{0}^{-1}[x, 1]\right.\right.$ for some $x \in[0,1] \vee\left(A=\pi_{0}^{-1}[0, x] \vee A=\pi_{0}^{-1}[x, m]\right.$ for some $\left.\left.x \in[0, m]\right)\right\}$

4- Fundamenta Mathematicae T. Cvir/3 
be the canonical binary normal subbase for $[0,1] \times[0, m]$. With the same technique as in [14] it follows that $\lambda\left(X, \mathscr{A}_{n}\right)$, where $\mathscr{A}_{n}=\{S \cap X \mid S \in \mathscr{P}\}$ (here $X$ refers to the embedded copy of $X$ in $[0,1] \times[0, m])$, is canonically homeomorphic to the convex-hull of $X$ in $[0,1] \times[0, m]$. Also, using the same technique as in [14], it follows that $\lambda\left(X, \bigcup_{n \in E} \mathscr{A}_{n}\right)$ can be embedded in $\prod_{n \in E} \lambda\left(X, \mathscr{A}_{n}\right)$ as an infinite dimensional compact linearly convex set. Hence $\lambda\left(X_{,} \bigcup_{n \in E} \mathscr{A}_{n}\right)$ is a Hilbert cube, by Keller's [12] theorem.

Now let $\mathscr{T}$ be a countable closed basis for $X$ which is closed under finite intersections and finite unions. The compactness of $X$ now implies that $\mathscr{T}$ separates the
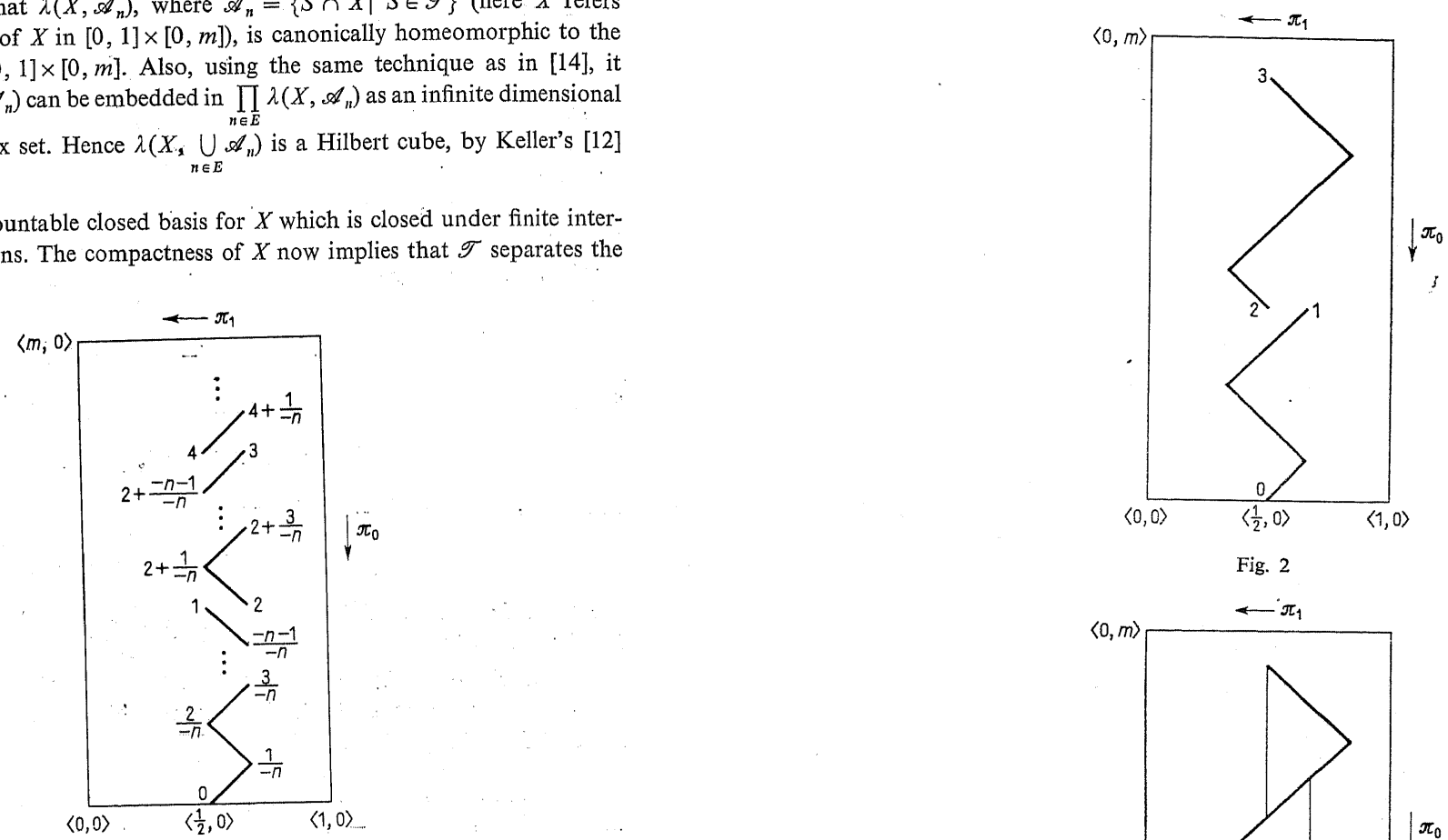

Fig. 2

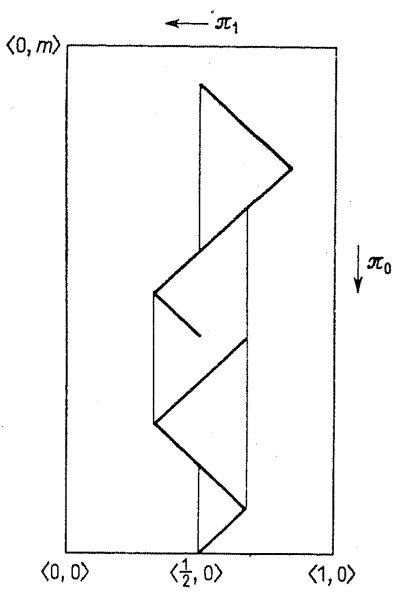

Fig. 3

and let $\left\{\left(T_{0}^{i}, T_{1}^{i}\right) \mid i \in N-\{1\}\right\}$ be an enumeration of $\mathscr{F}$. For each $i \in N-\{1\}$ it is geometrically clear that there is an embedding of $X$ in $[0,1] \times[0, m]$ of the type sketched in Figure 2 such that there is a $t \in[0,1]$ with the property that either $\left(\pi_{0}\left(T_{0}^{i}\right) \subset[0, t)\right)$ and $\left(\pi_{0}\left(T_{1}^{i}\right) \subset(t, 1]\right)$ or $\left(\pi_{0}\left(T_{1}^{i}\right) \subset[0, t)\right)$ and $\left(\pi_{0}\left(T_{0}^{i}\right) \subset(t, 1]\right)$

For sake of simplicity we have only indicated such an embedding for the case $m=3$. Define $\mathscr{S}_{n}:=\{S \cap X \mid S \in \mathscr{S}\}$ (here $X$ refers again to the embedded copy of $X$ in $[0,1] \times[0, m])$. As in [14] it is easy to show that $\lambda\left(X, \mathscr{S}_{n}\right)$ is canonically homeomorphic to the space indicated in Figure 3. 
Notice that $\lambda\left(X, \mathscr{S}_{n}\right)$ is connected.

Define $\mathscr{S}_{1}:=\bigcup_{n \in E} \mathscr{A}_{n}$, where the $\mathscr{A}_{n}$ 's are as defined above. Using precisely the same technique as in [14] Lemma 8, it can be shown that for each $n \in N$ the space $\lambda\left(X, \bigcup_{i=1}^{n} \mathscr{S}_{i}\right)$ is a $Q$-manifold, i.e. a separable metric space which admits an open covering by sets homeomorphic to open subsets of the Hilbert cube $Q$. In addition, $\lambda\left(X, \bigcup_{i=1}^{n} \mathscr{S}_{n}\right)$ is connected by Theoren 1.2 , and has a binary normal subbase since it is easily seen that $\bigcup_{i=1}^{n} \mathscr{S}_{i}$ is normal. Therefore $\lambda\left(X, \bigcup_{i=1}^{n} \mathscr{S}_{i}\right)$ is an AR by a theorem of van Mill [14]. This implies that $\lambda\left(X, \bigcup_{i=1}^{n} \mathscr{S}_{i}\right)$ is a Hilbert cube since each compact contractible $Q$-manifold is a Hilbert cube (cf. Chapman [3]).

It now is straightforward to prove that the inverse limit of the inverse sequence

$$
\lambda\left(X, \mathscr{S}_{1}\right) \stackrel{g_{1}}{\rightarrow} \lambda\left(X, \mathscr{S}_{2}\right) \stackrel{g_{2}}{\rightarrow} \lambda\left(X, \mathscr{S}_{3}\right) \leftarrow \ldots,
$$

with bonding maps $g_{i}: \lambda\left(X, \mathscr{S}_{i+1}\right) \rightarrow \lambda\left(X, \mathscr{S}_{i}\right)$ defined by

$$
g_{i}(\mathscr{M}):=\mathscr{M} \cap \mathscr{S}_{i}
$$

(cf. Section 2) is homemorphic to $\mu X$. (Define for each $i \in N$ a mapping $\xi_{i}: \mu X \rightarrow$ $\rightarrow \lambda\left(X, \mathscr{S}_{i}\right)$ by $\xi_{i}(\mathscr{M}):=\mathscr{M} \cap \mathscr{S}_{i}$. It is easily seen that $\xi_{i}=g_{i} \circ \xi_{i+1}$ for all $i \in N$. Hence the mapping $e: \mu X \rightarrow \underline{\lim }\left(\lambda\left(X, \mathscr{S}_{i}^{*}\right), g_{i}\right)$ defined by $e(\mathscr{M})_{i}=\xi_{i}(\mathscr{M})$ is continuous and onto. In addition $e$ is easily seen to be one to one, showing that $e$ is a homeomorphism).

In addition the mappings $g_{i}$ are $\mathrm{cp}$ mappings by Theorem 2.3. This implies that each point inverse of $g_{i}$ is an AR, being a retract of $\lambda\left(X, \mathscr{S}_{i+1}\right)$ (cf. Theorem 2.1(ii)). Consequently, $g_{i}$ is cellular (even in a strong way). It is a deep result of Chapman [4], [5] (cf. also Chapman [6]) that each cellular mapping between Hilbert cubes is a uniform limit of homeomorphisms (a so called near-homeomorphism). Hence we find that $\mu X$ is (homeomorphic to) the inverse limit of a sequence of Hilbert cubes with near-homeomorphisms as bonding maps. Applying results of Brown [2] (cf. also Mioduszewski [21]) we find that $\mu X$ is a Hilbert cube. This completes the proof of Theorem 4.1 .

4.1. THeOREM. Let $X$ be a finite sum of closed unit segments. Then $\mu X$ is a Hilbert cube.

5. Superextensions of finite graphs are Hilbert cubes. In this section we prove that if $X$ is a sum of finitely many nondegenerate finite connected graphs the space $\mu X$ is a Hilbert cube. This result is used in Section 6 to obtain the main result in this paper.

Let us recall the following theorem (cf. van Mill [16], Theorem 2.2.5).
5.1. Theorem. Let $\mathscr{S}$ be a binary subbase for the topological space $X$. Let $Y$ be a subspace of $X$ such. that for all $S_{0}, S_{1} \in \mathscr{S}$ with $S_{0} \cap S_{1} \neq \varnothing$ we have that also $S_{0} \cap S_{1} \cap Y \neq \varnothing$. Then $X$ is homeomorphic to $\lambda(Y, \mathscr{S} \uparrow Y)$, where $\mathscr{S} \uparrow Y$ $=\{S \cap Y \mid S \in \mathscr{P}\}$.

The proof of this theorem is very simple; indeed define a function $\varphi: X \rightarrow$ $\rightarrow \lambda(Y, \mathscr{S} \mid Y)$ by $\varphi(x):=\{S \cap Y \mid S \in \mathscr{S}$ and $x \in S\}$. It is straightforward to prove that $\varphi$ is a homeomorphism.

We are now prepared to prove the main result in this section.

5.2. THEOREM. Let $X$ be a sum of finitely many nondegenerate finite connected graphs. Then $\mu X$ is a Hilbert cube.

Proof. Let $F=\left\{x_{1}, \ldots, x_{n}\right\}$ be a finite subset of $X$ with the property that the components of $X-F$ are either homeomorphic to $(0,1)$ or to $[0,1)$. We denote the collection of components by $C_{1}, \ldots, C_{m}$. We may assume that $F$ does not contain any endpoint of some component of $X$. For each $i \leqslant m$ let $I_{i}^{k}(k \in N)$ be a sequence of arcs such that

$$
\begin{aligned}
& \text { (i) } k<l \Rightarrow I_{i}^{k} \subset I_{i}^{l} \text {; } \\
& \text { (ii) } \bigcup_{k=1}^{\infty} I_{i}^{k}=C_{i} .
\end{aligned}
$$

For each $k \in N$ define $X_{k}:=\bigcup_{i \leqslant m} I_{i}^{k}$. Clearly $\bigcup_{k=1}^{\infty} X_{k}$ is dense in $X$. We will show that $\left\{\mu X_{k} \mid k \in N\right\}$ with appropriate bonding mappings approximates $\mu X$. For each $k \in N$ and $i \leqslant m$ let $a_{i}^{k}$ and $b_{i}^{k}$ denote the endpoints of $I_{i}^{k}$. Define $\tilde{I}_{i}^{k}:=I^{k}-\left\{a_{i}^{k}, b_{i}^{k}\right\}$. For all $j \leqslant n$ let $D_{j}^{k}$ be the component of $X-\bigcup_{i \leqslant m} \hat{I}_{i}^{k}$ containing $x_{j}$. Then $D_{j}^{k}$ either is an interval, in case $x_{j}$ is a cutpoint $D_{j}^{k}$, or is a finite acyclic tree with precisely one branch point, namely $x_{j}$. In case $D_{j}^{k}$ is an interval, say with endpoints $c$ and $d$, it is obvious that there is an embedding $\varphi ; D_{j}^{k} \rightarrow\{c, d\}^{+} \subset \mu X_{k}$ such that $\varphi(c)=c$ and $\varphi(d)=d$ (notice that $\mu X_{k} \approx Q$ by Theorem 4.1 and that $\{c, d\}^{+}$is an AR by Theorem 2.1(ii) and hence that there is an arc in $\{c, d\}^{+}$connecting $c$ and $d$ ). In case $D_{j}^{k}$ is not an interval let $G \subset D_{j}^{k}$ be the (finite) set of endpoints of $D_{j}^{k}$. Then $|G| \geqslant 3$. Take a point $\mathscr{M} \in \mu X_{k}$ such that $\mathscr{M} \in \bigcap_{\theta \in G}(G-\{g\})^{+}$. There is such a point since $\{G-\{g\} \mid g \in G\}$ is linked. Now Lemma 3.2 implies that there is an embedding $\varphi: D_{j}^{k} \rightarrow G^{+} \subset \mu X_{k}$ such that $\varphi$ restricted to $G$ is the identity.

This procedure shows that for each $k \in N$ there is an embedding $\psi_{k}: X \rightarrow \mu X_{k}$ satisfying the following conditions:

(i) $\psi_{k}$ restricted to $X_{k}$ is the identity;

(ii) for all $i \leqslant n$ we have that $\psi_{k}\left(D_{i}^{k}\right) \subset\left(D_{i}^{k} \cap X_{k}\right)^{+} \cap \mu X_{k}$.

We now define other mappings from $X$ to $\mu X_{k}$. Indeed, fix $j \leqslant n$ and consider $D_{j}^{k}$ and $D_{j}^{k+1}$. Without loss of generality we may assume that $\left(D_{j}^{k+1} \cap X_{k+1}\right) \cap\left(D_{j}^{k} \cap X_{k}\right)$ $=\varnothing$ (for all $k \in N$ and $j \leqslant n$ ). Now, define a mapping $f_{k}: X \rightarrow \mu X_{k}$ by:

$$
f_{k}(x)=x \text { for all } x \in X_{k},
$$


$f_{k}$ restricted to $D_{j}^{k}-D_{j}^{k+1}$ is a homeomorphism of $D_{j}^{k}-D_{j}^{k+1}$ onto $\psi_{k}\left[D_{j}^{k}\right]-$ $-\left\{\psi_{k}\left(x_{j}\right)\right\}$,

$f_{k}$ maps $D_{j}^{k+1}$ onto $\psi_{k}\left(x_{j}\right)$.

It is clear that $f_{k}$ defined in this way is well defined and continuous. In addition, let $\xi_{k}: X_{k+1} \rightarrow \mu X_{k}$ be the restriction of $f_{k}$ to $X_{k+1}$. Then $\xi_{k}$ restricted to $X_{k}$ is the identity and consequently $X_{k} \subset \xi_{k}\left(X_{k+1}\right) \subset \mu X_{k}$. Define

$$
\mathscr{S}_{k}:=\left\{T^{+} \cap \xi_{k}\left(X_{k+1}\right) \mid T \in \mathscr{T}\left(X_{k}\right)\right\} .
$$

By Theorem 5.1 we conclude that $\lambda\left(\xi_{k}\left(X_{k+1}\right), \mathscr{S}_{k}\right)$ is canonically homeomorphic to $\mu X_{k}$. Also, it is easy to show that $\mathscr{S}_{k} \subset \mathscr{T}\left(\xi_{k}\left(X_{k+1}\right)\right)$ and that it is a super-normal subbase. Hence Theorem 2.3 shows that $\xi_{k}$ can be extended to a cp surjection $\mu\left(\xi_{k}\right): \mu X_{k+1} \rightarrow \lambda\left(\xi_{k}\left(X_{k+1}\right), \mathscr{S}_{k}\right)$. Let us identify $\mu X_{k}$ and $\lambda\left(\xi_{k}\left(X_{k+1}\right), \mathscr{S}_{k}\right)$. We then have the following diagram:

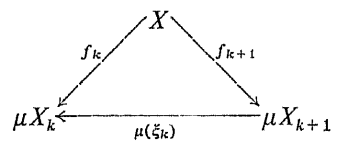

Claim. Diagram $(*)$ commutes.

Indeed, take $x \in X$. If $x \in X_{k+1}$ then

$$
\mu\left(\xi_{k}\right) f_{k+1}(x)=\mu\left(\xi_{k}\right)(x)=\xi_{k}(x)=f_{k}(x),
$$

hence there is no problem. Let us assume that $x \notin X_{k+1}$; then $x \in D_{j}^{k+1}$ for some $j \leqslant n$. Then $f_{k+1}(x) \in \psi_{k+1}(X)-X_{k+1}$. Hence, by construction of the embeddings $\psi_{k}$, we have that

$$
f_{k+1}(x) \in \psi_{k+1}\left(D_{j}^{k+1}\right) \subset\left(D_{j}^{k+1} \cap X_{k+1}\right)^{+} \subset \mu X_{k+1}
$$

and consequently, since $I\left(D_{j}^{k+1} \cap X_{k+1}\right)=\left(D_{j}^{k+1} \cap X_{k+1}\right)^{+}$, by Corollary 2.6,

$$
\mu\left(\xi_{k}\right) f_{k+1}(x) \in I\left(\xi_{k}\left(D_{j}^{k+1} \cap X_{k+1}\right)\right)=I\left(\left\{\psi_{k}\left(x_{j}\right)\right\}\right)=\left\{\psi_{k}\left(x_{j}\right)\right\} .
$$

This implies that $\mu\left(\xi_{k}\right) f_{k+1}(x)=f_{k}(x)$.

By a similar construction as above for each $k \in N$ we can extend the function $f_{k}: X \rightarrow \mu X_{k}$ to a cp surjection $\mu\left(f_{k}\right): \mu X \rightarrow \mu X_{k}$. Since diagram (*) commutes it is easy to verify that $\mu\left(f_{k}\right)=\mu\left(\xi_{k}\right) \circ \mu\left(f_{k+1}\right)$ for all $k \in N$ (this also follows from Theorem 2.3 since the composition of $\mathrm{cp}$ mappings is a $\mathrm{cp}$ mapping).

We conclude that the mapping $e: \mu X \rightarrow \varliminf\left(\mu X_{k}, \mu\left(\xi_{k}\right)\right)$ defined by $e(\mathscr{M})_{k}$ $:=\mu\left(f_{k}\right)(\mathscr{M})$ is a continuous surjection. We claim that $e$ is one to one, proving that it is a homeomorphism between $\mu X$ and $\underline{\lim }\left(\mu X_{k}, \mu\left(\xi_{k}\right)\right)$. Indeed, take distinct $\mathscr{M}, \mathscr{N} \in \mu X$ and take $M \in \mathscr{M}$ and $N \in \mathscr{N}$ such that $M \cap N=\varnothing$. Then $(M \cap F) \cap$ $\cap(N \cap F)=\varnothing$. Let $M \cap F=\left\{x_{1}, \ldots, x_{i}\right\}$ and $N \cap F=\left\{x_{i+1}, \ldots, x_{n}\right\}$ (if $M \cup N \ngtr F$ then enlarge $M$ with the set $F-(M \cup N)$, then $M$ still belongs to $\mathscr{M}$ and also $M$ does not intersect $N)$. Let $U_{i}(i \leqslant n)$ be open subsets of $X$ such that $x_{i} \in U_{i}$ and $i<j$ implies that $U_{i} \cap U_{j}=\varnothing$ while in addition $\bigcup_{j \leqslant i} U_{j} \subset X-N$ and $\bigcup_{j=i+1}^{n} U_{i}$ $\subset X-M$. Choose $k \in N$ such that $D_{i}^{k} \subset U_{i}$ for all $i \leqslant n$. Then

and

$$
\mu\left(f_{k}\right)(\mathscr{M}) \in\left(\left(M \cap X_{k}\right) \cup\left(X_{k} \cap \bigcup_{j=1}^{i} D_{j}^{k}\right)\right)^{+}
$$

$$
\mu\left(f_{k}\right)(\mathcal{N}) \in\left(\left(N \cap X_{k}\right) \cup\left(X_{k} \cap \bigcup_{j=i+1}^{n} D_{j}^{k}\right)\right)^{+},
$$

and as these sets are disjoint, we conclude that $\mu\left(f_{k}\right)(\mathscr{M}) \neq \mu\left(f_{k}\right)(\mathscr{N})$. This shows that $\mu X \approx \varliminf\left(\mu X_{k}, \mu\left(\xi_{k}\right)\right)$. By Theorem 4.1 and by the cellularity of the mappings $\mu\left(\xi_{k}\right)$ (cf. Theorem 2.3) we conclude, with similar arguments as in Section 4 , that $\mu X \approx Q$.

6. Superextensions of metrizable continua are Hilbert cubes. In this final section we prove that $\mu X$ is a Hilbert cube provided that $X$ is a finite sum of nondegenerate metrizable continua. We heavily rely on results of Curtis and Schori [9], [10].

As noted in Section 2 for compact Hausdorff $X$ we may regard $\lambda X$ to be a subspace of $2^{2^{x}}$. Now let $(X, d)$ be a compact metric space and let $d_{H}$ (resp. $\left.d_{H H}\right)$ be the Hausdorff metric for $2^{X}$ (resp. $2^{2^{x}}$ ). Let $\mathscr{L}(X) \subset 2^{2^{X}}$ denote the space of all closed families of nonvoid closed subsets of $X$, which are linked (cf. van Mill and Van de Vel [20]). In van Mill and Van de Vel [20] the following results were established.

6.1. TheOREM. (i) The inclusion $(\lambda X, \bar{d}) \rightarrow\left(2^{2^{x}}, d_{H H}\right)$ is an isometry (here $\bar{d}$ is Verbeek's [27] metric for $\lambda X$, see Section 3);

(ii) For each $x \in X$ the mapping $h_{x}: \mathscr{L}(X) \rightarrow \lambda X$ defined by $h_{x}(\mathscr{L}):=p\left(x, \cap L^{+}\right)$ is a contractive retraction (with respect to the metrics $d_{H H}$ and $\bar{d}$ ).

Before proving the main result in this section we mention a highly nontrivial result of Curtis and Schori [10] (in fact Curtis and Schori proved a stronger assertion than stated below, see [10]).

6.2. THEOREM. Let $X$ be a nondegenerate Peano continuum. Then there is collection finite connected graphs $\left\{\Gamma_{i} \mid i \in N\right\}$ in $X$ and for each $i \in N$ there is a mapping $\varphi_{i}: \Gamma_{i+1} \rightarrow 2^{\Gamma_{i}}$ such that

(i) $\Gamma_{i} \rightarrow X$ (in $2^{X}$ );

(ii) if $f_{i}: 2^{r_{i+1}} \rightarrow 2^{r_{i}}$ is the mapping induced by $\varphi_{i}$, i.e. $f_{i}(A)=\bigcup\left\{\varphi_{i}(a) \mid a \in A\right\}$, then $\sum_{i=1}^{\infty} d_{H}\left(f_{i}\right.$, id $)<\infty$;

(iii) $\left\{f_{i} \circ \ldots \circ f_{j} \mid j \geqslant i\right\}$ is an equi-uniformly continuous family for each $i$ (iv) each $f_{i}$ is onto.

Notice that since the $f_{i}$ 's are onto for every $y \in \Gamma_{i}$ there is an $x \in \Gamma_{i+1}$ such that $\varphi_{i}(x)=y$. 
Actually, Curtis and Schori, proved Theorem 6.2 in [9] for compact connected polyhedra and in [10] they proved the general case. In our construction we need the result for polyhedra only. It must be noticed that the proof of 6.2 for polyhedra is easier than the proof of the general case.

6.3. Proposition. Let $X$ be a finite sum of nondegenerate compact connected polyhedra. Then $\mu X$ is a Hilbert cube.

Proof. Let $P_{1}, \ldots, P_{n}$ denote the collection of components of $X$. For each $i \leqslant n$ let $\Gamma_{j}^{i}$ and $\varphi_{j}^{i}(j \in N)$ be a collection of finite graphs and mappings with properties as in Theorem 6.2. For each $j \in N$ define $X_{j}:=\bigcup_{i \leq n} \Gamma_{j}^{i}$; in addition define $\varphi_{j}^{*}: X_{j+1} \rightarrow 2^{X_{j}}$ by $\varphi_{j}^{*}(x)=\varphi_{j}^{i}(x)$ if $x \in \Gamma_{j}^{i}$. It is clear that the $\varphi_{j}^{*}$ defined in this way are continuous. Let $f_{j}^{*}: 2^{X_{j+1}} \rightarrow 2^{X_{j}}$ be the mapping induced by $\varphi_{j}^{*}$ (i.e. $\left.f_{j}^{*}(A)=\bigcup \varphi_{j}^{*}[A]\right)$. In addition, let $F_{j}^{*}: 2^{2^{X_{j+1}}} \rightarrow 2^{X_{j}}$ be the mapping induced by $f_{j}^{*}$ (i.e. $F_{j}^{*}(\mathscr{A})$ $\left.=\left\{f_{j}^{*}(A) \mid A \in \mathscr{A}\right\}\right)$. Fix $j \in N$. Take $\mathscr{A} \in \mathscr{L}\left(X_{j+1}\right)$. We claim that $F_{j}^{*}(\mathscr{A}) \in \mathscr{L}\left(X_{j}\right)$. Indeed, take $A_{0}, A_{1} \in \mathscr{A}$. Then $A_{0} \cap A_{1} \neq \varnothing$ implies that $\varphi_{j}^{*}\left[A_{0}\right] \cap \varphi_{j}^{*}\left[A_{1}\right] \neq \varnothing$ Take $C \in \varphi_{j}^{*}\left[A_{0}\right] \cap \varphi_{j}^{*}\left[A_{1}\right]$. Then $\varnothing \neq C \subset \cup \varphi_{j}^{*}\left[A_{0}\right] \cap \cup \varphi_{j}^{*}\left[A_{1}\right]$. We conclude that $f_{j}^{*}\left(A_{0}\right) \cap f_{j}^{*}\left(A_{1}\right) \neq \varnothing$ and consequently $F_{j}^{*}(\mathscr{A}) \in \mathscr{L}\left(X_{j}\right)$. By induction, choose for every $j \in N$ a point $x_{j} \in X_{j}$ such that $\varphi_{j}^{*}\left(x_{j+1}\right)=x_{j}$. This can be done (cf. the remark following Theorem 6.2). Now consider the following infinite diagram.

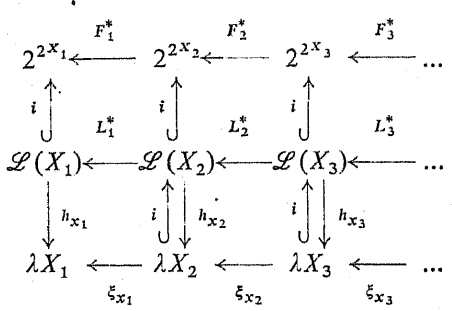

where $L_{j}^{*}$ is the restriction of $F_{j}^{*}$ to $\mathscr{L}\left(X_{j+1}\right)$ and $i$ always denotes inclusion and $h_{x_{j}}$ is the mapping of Theorem 6.1(ii) (i.e. $h_{x_{j}}(\mathscr{M})=p\left(x_{j}, \bigcap_{M \in \mathscr{M}} M^{+}\right)$) and $\xi_{x_{j}}$ is the mapping of Theorem 2.2 (i.e. $\zeta_{x_{j}}(\mathscr{A})=p\left(x_{j}, \bigcap_{M \in \mathcal{M}}\left(\bigcup \varphi_{j}^{*}[M \in)^{+}\right)\right)$.

Claim 1. Each $\xi_{x_{j}}$ is onto.

Indeed, take $\mathscr{M} \in \lambda X_{j}$ and consider the collection $\left\{\varphi_{j}^{*-1}[M] \mid M \in \mathscr{M}\right\}$. This collection, which is a linked system of course, (cf. the remark following Theorem 6.2), can be extended to a point $\mathscr{N} \in \lambda X_{j+1}$. We claim that $\xi_{x j}(\mathscr{N})=\mathscr{M}$. Indeed, since $\left\{\varphi^{*-1}[M] \mid M \in \mathscr{M}\right] \subset \mathscr{N}$ we have that $\mathscr{H} \subset\left\{\cup \varphi_{j}^{*}[N] \mid N \in \mathscr{N}\right\}$ and as $\mathscr{H}$ is a maximal linked system we conclude that $\mathscr{M}=\left\{U \varphi_{j}^{*}[N] \mid N \in \mathscr{N}\right\}$. Hence

$$
\begin{aligned}
\xi_{j}(\mathscr{N}) & =p\left(x_{j}, \bigcap_{N \in \mathscr{N}}\left(\bigcup \varphi_{j}^{*}[N]\right)^{+}\right)=p\left(x_{j}, \bigcap_{M \in \mathscr{M}} M^{+}\right) \\
& =p\left(x_{j},\{\mathscr{M}\}\right)=\mathscr{A} .
\end{aligned}
$$

This proves the claim.

The following claim is more difficult to prove.

Claim 2. Suppose that $k<l$. Then

$$
h_{x_{k}} \circ L_{k}^{*} \circ L_{k+1}^{*} \circ \ldots \circ L_{l-1}^{*} \circ i=\xi_{x_{k}} \circ \xi_{x_{k+1}} \circ \ldots \circ \xi_{x_{l-1}} \text {. }
$$

We will only prove this for the case $k=1$ and $l=3$. The proof of the general case is identical (notice that the case $k=1$ and $l=2$ is trivial, because of the definitions of $L_{1}^{*}, h_{x_{1}}$ and $\left.\xi_{x_{1}}\right)$. Indeed, take $\mathscr{M} \in \lambda X_{3}$. It is easily verified that $L_{2}^{*} L_{1}^{*}(\mathscr{M})$ equals the collection $\left\{\bigcup \varphi_{1}^{*}\left[\cup \varphi_{2}^{*}[M]\right] \mid M \in \mathscr{M}\right\}$. Let this collection be denoted by $\mathscr{A}$. Notice that $\mathscr{A} \subset 2^{X}$ is closed. We claim that $p\left(x_{1}, \bigcap_{A \in \&} A^{+}\right)$, which equals $h_{x_{1}} L_{2}^{*} L_{1}^{*}(\mathscr{H})$, is the collection

$$
\left\{B \in 2^{X} \mid x_{1} \in B \text { and } B \cap A \neq \varnothing(\forall A \in \mathscr{A})\right\} \cup\left\{B \in 2^{X} \mid \exists A \in \mathscr{A}: A \subset B\right\} .
$$

This follows from the following general lemma which is of independent interest.

6.4. Lemma. Let $X$ be a compact Hausdorff space, let $x \in X$ and let $\mathscr{A} \subset 2^{X}$ be a closed (in $\left.2^{X}\right)$ and linked system. Then $p\left(x, \bigcap_{A \in \mathcal{A}} A^{+}\right)$equals the collection

$$
\left\{B \in 2^{X} \mid x \in B \text { and } B \cap A \neq \varnothing(\forall A \in \mathscr{A})\right\} \cup\left\{B \in 2^{X} \mid \exists A \in \mathscr{A}: A \subset B\right\} \text {. }
$$

Proof. In van Mill [15] Theorem 2.2 it was shown that the above collection is a pre-mls for $p\left(x, \bigcap_{A \in s t} A^{+}\right)$, that is, a linked subfamily of $p\left(x, \bigcap_{A \in \mathscr{A}^{+}} A^{+}\right)$which is contained in precisely one mls, namely $p\left(x, \cap A^{+}\right)$.

For each nonvoid collection $\mathscr{C} \subset 2^{X}$ define

and

$$
C^{\uparrow}:=\left\{B \in 2^{X} \mid \exists C \in \mathscr{C}: C \subset B\right\}
$$

$$
\mathscr{C}^{\perp}:=\left\{B \in 2^{X} \mid \forall C \in \mathscr{C}: C \cap B \neq \varnothing\right\}
$$

respectively. In van Mill and Van de Vel [20] $\mathscr{C}^{\perp}$ was called the collection of transversal sets of $\mathscr{C}$. It is easily seen that $\mathscr{C}^{\perp}$ is always closed in $2^{x}$ and that $\mathscr{C}^{\uparrow}$ is closed provided that $\mathscr{C}$ is closed (use the compactness of $2^{X}$ ).

We conclude that the collection

$\left\{B \in 2^{X} \mid x \in B\right.$ and $B \cap A \neq \varnothing$ for all $\left.A \in \mathscr{A}\right\} \cup\left\{B \in 2^{X} \mid \exists A \in \mathscr{A}: A \subset B\right\}$ equals $\mathscr{W}^{\dagger} \cup(\mathscr{A} \cup\{x\})^{\perp}$ and hence is closed in $2^{X}$.

Now assume that there is a point $C \in p\left(x, \bigcap_{A \in \mathscr{A}} A^{+}\right)-\left(\mathscr{A}^{\dagger} \cup(\mathscr{A} \cup\{x\})^{\perp}\right)$. Let $U_{1}, \ldots, U_{n} \subset X$ be open such that $C \subset \bigcup_{i \leqslant n} U_{i}$ and $C \cap U_{i} \neq \varnothing$ for all $i \leqslant n$ while in addition $\left\langle\operatorname{cl}_{X}\left(U_{1}\right), \ldots, \operatorname{cl}_{X}\left(U_{n}\right)\right\rangle \cap\left(\mathscr{A}^{\dagger} \cup(\mathscr{A} \cup\{x\})^{\perp}\right)=\varnothing$. If $\bigcup_{i \leqslant n} U_{i}$ does not contain a member of $\left(\mathscr{A}^{\dagger} \cup(\mathscr{A} \cup\{x\})^{\perp}\right)$ we conclude that $X-\bigcup_{i \leqslant n} U_{i} \in p\left(x, \bigcap_{A \in \mathscr{A}} A^{+}\right)$ since $\left(\mathscr{A}^{\uparrow} \cup(\mathscr{A} \cup\{x\})^{\perp}\right)$ is a pre-mls for $p\left(x, \bigcap_{A \in \mathscr{A}} A^{+}\right)$. This contradicts 
$C \cap\left(X-\bigcup_{i \leqslant n} U_{i}\right)=\varnothing$. Therefore $\bigcup_{i \leqslant n} U_{i}$ contains an element of $\mathscr{A}^{\uparrow} \cup(\mathscr{A} \cup\{x\})^{\perp}$; consequently, since $\left(\mathscr{A}^{\uparrow} \cup(\mathscr{A} \cup\{x\})^{\perp}\right)^{\uparrow}=\mathscr{A}^{\uparrow} \cup(\mathscr{A} \cup\{x\})^{\perp}$, we find that $\cup \mathrm{cl}_{X}\left(U_{i}\right) \in \mathscr{A}^{\dagger} \cup(\mathscr{A} \cup\{x\})^{\perp}$ which is a contradiction since $\cup \operatorname{cl}_{X}\left(U_{i}\right)$ $\in\left\langle\mathrm{cl}_{X}\left(U_{1}\right), \ldots, \mathrm{cl}_{X}\left(U_{n}\right)\right\rangle$.

This proves that $p\left(x_{1}, \bigcap_{A \in \mathscr{A}} A^{+}\right)=\mathscr{A}^{\uparrow} \cup(\mathscr{A} \cup\{x\})^{\perp}$ (the notation is as in the proof of Lemma 6.4).

We will now calculate $\xi_{x_{1}} \xi_{x_{2}}(\mathscr{M})$. Define $\mathscr{B}:=\left\{\bigcup \varphi_{2}^{*}[M] \mid M \in \mathscr{M}\right\}$. Then, by Lemma $6.4, \xi_{x_{2}}(\mathscr{M})$ equals $\mathscr{B}^{\uparrow} \cup\left(\mathscr{B} \cup\left\{x_{2}\right\}\right)^{\perp}$. Clearly $\left\{\cup \varphi_{1}^{*}[B] \mid B \in \mathscr{B}\right\}$ equals $\mathscr{A}$. In addition, if $E \in\left(\mathscr{B} \cup\left\{x_{2}\right\}\right)^{\perp}$ then, since $\xi_{x_{1}}\left(x_{2}\right)=x_{1}$, we find that $\bigcup \varphi_{1}^{*}[E]$ contains $x_{1}$ and intersects all members from $\mathscr{A}$. Therefore $\xi_{x_{1}} \xi_{x_{2}}(\mathscr{M})$ and $p\left(x, \cap A^{+}\right)$are both the union of $\mathscr{A}^{\dagger}$ and a set consisting of closed sets which all contain $x_{1}$ and which all meet every member from $\mathscr{A}$. As $\xi_{x_{1}} \xi_{x_{2}}(\mathscr{M})$ and $p\left(x, \cap A^{+}\right)$ are maximal linked systems they are equal. This proves Claim 2.

CLAIM 3. $\left\{\xi_{x_{i}} \circ \ldots \circ \dot{\xi}_{x_{j}} \mid j \geqslant i\right\}$ is an equi-uniformly continuous family for each $i$.

It is clear that there is a metric $d$ for $X$ such that $\left\{f_{i}^{*} \circ \ldots \circ f_{j}^{*} \mid j \geqslant i\right\}$ is an equiuniformly continuous family for each $i$. For this metric we also have that $\sum_{i=1}^{\infty} d_{H}\left(f_{i}^{*}\right.$, id $)<\infty$. A straightforward check now shows that $\left\{F_{i}^{*} \circ \ldots \circ F_{j}^{*} \mid j \geqslant i\right\}$ is an equi-uniformly continuous family for each $i$. This implies that $\left\{L_{i}^{*} \circ \ldots \circ L_{j}^{*} \mid j \geqslant i\right\}$ is an equi-uniformly continuous family for each $i$. By Claim 2 and by the fact that the $h_{x_{i}}$ 's are metric contractions (cf. Theorem 6.1(ii)) it follows that $\left\{\xi_{x_{i}} \circ \ldots \circ \xi_{x_{j}} \mid j \geqslant i\right\}$ is equi-uniformly continuous for each $i$.

For each $i \in N$ the set $\lambda X_{i} \subset 2^{2^{x_{i}}} \subset 2^{2^{x}}$. However $\lambda X_{i}$ is not a subspace of $\lambda X$, although it is easy to define a canonical embedding $\eta_{i}: \lambda X_{i} \rightarrow \lambda X$. Indeed, define $\eta_{i}(\mathscr{M}):=\left\{A \in 2^{X} \mid A \cap X_{i} \in \mathscr{M}\right\}$. Notice that $\mathscr{M} \subset \eta_{i}(\mathscr{M})$. It is easily seen that this defines an embedding of $\lambda X_{i}$ into $\lambda X$ which in addition does not change distance, i.e.

$$
d_{H H}(\mathscr{M}, \mathscr{N})=d_{H H}\left(\eta_{i}(\mathscr{M}), \eta_{i}(\mathscr{N})\right) \quad \text { for all } \mathscr{M}, \mathscr{N} \in \lambda X_{i}
$$

The set $\eta_{i}\left[\lambda X_{i}\right]$ will be denoted by $\lambda^{*} X_{i}$. Define $g_{i}: \lambda^{*} X_{i+1} \rightarrow \lambda^{*} X_{i}$ as the composition $\eta_{i} \circ \xi_{x_{i}} \circ \eta_{i+1}^{-1}$. Since the $\eta_{i}$ 's do not change distances Claim 3 implies that $\left\{g_{i} \circ \ldots \circ g_{j} \mid j \geqslant i\right\}$ is an equi-uniformly continuous family for each $i \in N$.

ClaIM 4. $\bar{d}\left(g_{i}, \mathrm{id}\right) \leqslant d_{H}\left(f_{i}^{*}, \mathrm{id}\right)$ for each $i$.

Indeed take $\mathscr{M} \in \lambda^{*} X_{i+1}$ and let $\mathscr{A}:=\left\{M \cap X_{i+1} \mid M \in \mathscr{M}\right\}$. Then by the definition $\xi_{x_{i}}$, we have that $g_{i}(\mathscr{M})$ contains the family $\left\{\bigcup \varphi_{i}^{*}[A] \mid A \in \mathscr{A}\right\}$. Let $\delta:=d_{H}\left(f_{i}^{*}\right.$, id $)$. Now take $M \in \mathscr{M}$ and let $A:=M \cap X_{i+1}$. Then $d_{H}\left(A, \cup \varphi_{i}^{*}[A]\right) \leqslant \delta$ which implies that $\bigcup \varphi_{i}^{*}[A] \subset B_{\delta}(A) \subset B_{\delta}(M)$. Therefore, since $\bigcup \varphi_{i}^{*}[A] \in g_{i}(\mathscr{M})$ we conclude that $B_{\delta}(M) \in \mathscr{M}$. Consequently, $\partial\left(\mathscr{M}, g_{i}(\mathscr{M})\right) \leqslant \delta$ by expression 3 of Section 3. Hence $\bar{d}\left(g_{i}, \mathrm{id}\right) \leqslant d_{H}\left(f_{i}^{*}, \mathrm{id}\right)$.
This implies that $\sum_{i=1}^{\infty} \bar{d}\left(g_{i}, \mathrm{id}\right) \leqslant \sum_{i=1}^{\infty} d_{H}\left(f_{i}^{*}, \mathrm{id}\right)<\infty$. In addition, since $X_{i} \rightarrow X$ (in $2^{X}$ ) it is easily seen that $\lambda^{*} X_{i} \rightarrow \lambda X\left(\right.$ in $\left.2^{\lambda X}\right)$. We conclude that $\lambda X \approx \underline{\lim }\left(\lambda^{*} X_{i}, g_{i}\right)$. This can be seen as follows; denote $\varliminf\left(\lambda^{*} X_{i}, g_{i}\right)$ by $Y$. For each $\left\langle y_{i}\right\rangle_{i} \in Y$ the sequence $\left\langle y_{i}\right\rangle_{i}$ is Cauchy in $\lambda X$ by the fact that $\sum_{i=1}^{\infty} \bar{d}\left(g_{i}\right.$, id $)<\infty$, and hence converges to some point $y \in \lambda X$. The mapping $e: Y \rightarrow \lambda X$ defined by $e\left(\left\langle y_{i}\right\rangle_{i}\right)=\lim _{i \rightarrow \infty} y_{i}$ is easily seen to be continuous, is one to one since $\left\{g_{i} \circ \ldots \circ g_{j} \mid j \geqslant i\right\}$ is an equiuniformly continuous family for each $i$, and is onto since $\lambda^{*} X_{i} \rightarrow \lambda X(i \rightarrow \infty)$. Of course this kind of argumentation is known (cf. Brown [2], Mioduszewski [21], Curtis and Schori [9]). Identify $\varliminf_{(1 m}\left(\lambda^{*} X_{i}, g_{i}\right)$ and $\lambda X$ and let $\pi_{i}: \lambda X \rightarrow \lambda^{*} X_{i}$ be the natural projection of $\lambda X$ onto $\lambda^{*} X_{i}(i \in N)$. Identify $\lambda^{*} X_{i}$ and $\lambda X_{i}(i \in N)$. For each $i \in N$ let $\varrho_{i}: \lambda X_{i} \rightarrow \mu X_{i}$ be the natural Jensen surjection of $\lambda X_{i}$ onto $\mu X_{i}$ (cf. Section 2 and in particular Theorem 2.3).

Clalm 5. The function $\beta_{i}: \mu X_{i+1} \rightarrow \mu X_{i}$ defined by $\beta_{i}:=\varrho_{i} \circ g_{i} \circ \varrho_{i+1}^{-1}$ is a well defined cp surjection.

Indeed, take $\mathscr{M} \in \mu X_{i+1}$ and assume that $\beta_{i}(\mathscr{M})$ contains two distinct points $\mathscr{P}_{0}$ and $\mathscr{P}_{1}$. Take $T_{0}, T_{1} \in \mathscr{T}\left(X_{i+1}\right)$ such that $T_{i} \in \mathscr{P}_{i}$ and $T_{0} \cap T_{1}=\varnothing$. It is clear that we may choose the $T_{i}$ 's in such a way that for each component $C$ of $X_{i+1}$ we have that $C$ is either contained in one of the $T_{i}$ 's or intersects both $T_{0}$ and $T_{1}$. Let $D$ be a component of $\lambda X_{i}$ such that $D \cap \varrho_{i}^{-1}\left(T_{0}^{+}\right)=\varnothing$. By the remarks in Section 3 we may assume that there is a finite family of clopen subsets $\mathscr{E}$ in $X_{i}$ such that $D=\bigcap_{E \in \mathscr{E}} E^{+}$. By the fact that $\varrho_{i}$ is a cp mapping (cf. Theorem 2.3) and the binarity of $\left\{A^{+} \mid A \in 2^{X_{i}}\right\}$ we may assume that there is a $E_{0} \in \mathscr{E}$ such that $E_{0}^{+} \cap \varrho_{i}^{-1}\left(T_{0}^{+}\right)=\varnothing$ (notice that the first "plus" is in $\lambda X$, while the second is in $\left.\mu X_{i+1}\right)$. Hence $E_{0} \subset \varrho_{i}\left[E_{0}^{+}\right]$does not intersect $T_{0}$. Consequently $E_{0} \subset T_{1}$ which implies that $E_{0} \subset \varrho_{i}^{-1}\left[T_{1}^{+}\right]$and by the fact that $\varrho_{i}$ is a cp mapping we see that $E_{0}^{+}=I\left(E_{0}\right) \subset \varrho_{i}^{-1}\left[T_{1}^{+}\right]$. This procedure shows that every component of $\lambda X_{i+1}$ is either contained in one of $\left\{\varrho_{i}^{-1}\left[T_{0}^{+}\right], \varrho_{i}^{-1}\left[T_{1}^{+}\right]\right\}$ or intersects both $\varrho_{i}^{-1}\left[T_{0}^{+}\right]$and $\varrho_{i}^{-1}\left[T_{1}^{+}\right]$. Now, since each component of $\lambda X_{i+1}$ is mapped by $g_{i}$ in a component of $\lambda X_{i}$ this implies that each component of $\lambda X_{i+1}$ is either contained in one of $\left\{g_{i}^{-1} \varrho_{i}^{-1}\left[T_{0}^{+}\right], g_{i}^{-1} \varrho_{i}^{-1}\left[T_{1}^{+}\right]\right\}$or intersects both $g_{i}^{-1} \varrho_{i}^{-1}\left[T_{0}^{+}\right]$and $g_{i}^{-1} \varrho_{i}^{-1}\left[T_{1}^{+}\right]$. For simplicity of notation write $V_{j}=g_{i}^{-1} \varrho_{i}^{-1}\left[T_{j}^{+}\right]$ $(j \in\{0,1\})$.

By the binarity of $\left\{A^{+} \mid A \in 2^{X_{i+1}}\right\}$ and by the fact that $\varrho_{i} \circ g_{i}$ is a cp mapping, there are disjoint $A_{j} \in 2^{X_{i+1}}(j \in\{0,1\})$ such that $V_{j} \subset A_{j}^{+}$. If $A_{0}$ is clopen then so is $A_{0}^{+}$and consequently $V_{0}=A_{0}^{+}$since each component of $\lambda X_{i+1}$ either is contained in one of $\left\{V_{0}, V_{1}\right\}$ or intersects both $V_{0}$ and $V_{1}$. Therefore $T_{0}^{+}$is a proper nonvoid clopen subset of $\mu X_{i}$, since $\varrho_{i} \circ g_{i}$ is an identification. This contradicts the connectedness of $\mu X_{i}$ (cf. Corollary 1.3). In the same way we have that $A_{1}$ is not a clopen subset of $X_{i+1}$. Hence $\left\{A_{0}, A_{1}\right\} \subset \mathscr{T}\left(X_{i+1}\right)$. Since $\left(\varrho_{i} \circ g_{i}\right)^{-1}\left(\mathscr{P}_{j}\right) \subset A_{j}^{+}(j \in\{0,1\})$ we see that $A_{0}$ and $A_{1}$ must be both elements of $\mathscr{M}$ (cf. Section 2); this contradicts the linkedness of $\mathscr{M}$. 
This proves that $\beta_{i}$ is well defined. Also, clearly $\beta_{i}$ is continuous. That $\beta_{i}$ is a cp mapping now follows from the fact that $\varrho_{i+1}, g_{i}$ and $\varrho_{i}$ are cp mappings and from Theorem 2.4 .

One moment one might think that this finishes the proof of the proposition since it is intuitively clear that the inverse sequence

$$
\mu X_{1} \stackrel{\beta_{1}}{\rightarrow} \mu X_{2} \stackrel{\beta_{2}}{\rightarrow} \mu X_{3} \leftarrow \ldots
$$

approximates $\mu X$. We will prove below that this is indeed the case, but since there is no canonical embedding of $\mu X_{i}$ in $\mu X$ we cannot apply similar arguments then in the $\lambda X$ case above. This causes a few technical problems.

Claim 6. For each $i \in N$ the projection $\pi_{i}: \lambda X \rightarrow \lambda X_{i}$ is a cp surjection.

For each $j>i$ let $g_{j i}: \lambda X_{j} \rightarrow \lambda X_{i}$ be the composition $g_{i} \circ \ldots \circ g_{j-1}$. In addition, let $g_{i i}$ be the identity mapping on $\lambda X_{i}$. Notice that we have identified the $\lambda X_{i}$ 's with subspaces of $\lambda X$. Now using the properties of the functions $g_{i}$ (namely $\sum_{i=1}^{\infty} d\left(g_{i}\right.$, id) $<\infty$ and the equi-uniform continuity) it is easy to calculate that for each closed set $A \subset \lambda X_{i}$ we have that

$$
\pi_{i}^{-1}(A)=\mathrm{cl}_{\lambda X}\left(\bigcup_{j \geqslant i} g_{j i}^{-1}(A)\right)
$$

Now let $T$ be a closed subset of $X_{i}$. For each $j \geqslant i$ define $E_{j}:=g_{j i}^{-1}\left(T^{+}\right)$. Notice that $E_{i}=T^{+}$. In addition, for each $j \geqslant i$ let $p_{j}$ be the nearest point mapping of $\lambda X_{j}$ (cf. Theorem 2.1(ii)). Now take a point $\mathscr{M} \in \lambda X-\pi_{i}^{-1}\left(T^{+}\right)$. Let $\mathscr{M}_{j}:=\pi_{j}(\mathscr{H})$ and $\mathcal{N}_{j}:=p_{j}\left(\mathscr{M}_{j}, E_{j}\right)$ (notice that the $g_{j i}$ 's are cp mappings). By Corollary 2.5 we have that $\left\{\mathcal{N}_{j} \mid j \geqslant i\right\}$ determines a thread in

$$
\lambda X_{i} \stackrel{g_{i}}{\leftarrow} \lambda X_{i+1} \stackrel{g_{i+1}}{\leftarrow} \ldots
$$

and hence a point, say $\mathcal{N}$, in $\lambda X$. By the fact that $\left\{g_{j} \circ \ldots \circ g_{i} \mid j \geqslant i\right\}$ is an equiuniformly continuous family for all $j \geqslant i$ we have that $\delta=\inf \left\{d\left(\mathscr{M}_{j}, \mathscr{N}_{j}\right) \mid j \geqslant i\right\}>0$. This implies that $\bar{d}(\mathscr{M}, \mathscr{N}) \geqslant \delta$. Hence we may take $M \in \mathscr{N}$ such that $B_{\delta / 2}(M) \notin \mathscr{N}$ (cf. Section 3, Expression 3). Choose $k \geqslant i$ such that $\mathscr{M}_{l} \in B_{\delta / 2}(M)$ for all $l \geqslant k$. Assume that there exists an $l \geqslant k$ such that $E_{l} \cap\left(U_{\delta / 2}(M)\right)^{+} \neq \varnothing$. Take a point $\mathscr{A} \in E_{l} \cap\left(U_{\delta / 2}(M)\right)^{+}$. Choose an index $l_{0} \geqslant l$ such that $\pi_{l_{1}}(\mathscr{A})=\mathscr{A}_{l_{1}} \in\left(U_{\delta / 2}(M)\right)^{+}$ for all $l_{1} \geqslant l_{0}$. Now consider $l_{0}$. We have that $\mathscr{A}_{l_{0}} \in E_{l_{0}}, \mathscr{N}_{l_{0}} \in E_{l_{0}}, p_{l_{0}}\left(\mathscr{M}_{l_{0}}, E_{l_{0}}\right)=\mathscr{N}_{l_{0}}$ and $d\left(\mathscr{A}_{l_{0}}, \mathscr{M}_{l_{0}}\right) \leqslant \frac{1}{2} \delta<\delta=d\left(\mathscr{N}_{l_{0}}, \mathscr{M}_{l_{0}}\right)$. This contradicts a theorem in van Mill and Van de Vel [19]; there it was shown that for each compact metric space $(X, d)$ the nearest point retraction $p: \lambda X \rightarrow A$, where $A$ is $\left(2^{X}\right)^{+}$-closed, is a metric nearest point map, in the sense that $d(\mathscr{M}, A)=d(\mathscr{M}, p(\mathscr{M}, A))$ for all $\mathscr{M} \in \lambda X$.

We conclude that $E_{l} \cap\left(U_{\delta / 2}(M)\right)^{+}=\varnothing$ for all $l \geqslant k$. Therefore we have that $E_{l} \subset\left(X-U_{\delta / 2}(M)\right)^{+}$for all $l \geqslant k$, and consequently,

$$
\pi_{i}^{-1}\left(T^{+}\right)=\pi_{k}^{-1}\left(E_{k}\right)=\operatorname{cl}_{\lambda X}\left(\bigcup_{l \geqslant k} E_{l}\right) \subset\left(X-U_{\delta / 2}(M)\right)^{+} .
$$

For each $\mathscr{H} \in \lambda X-\pi_{i}^{-1}\left(T^{+}\right)$we have constructed a closed set $B \subset X$ such that $\pi_{i}^{-1}\left(T^{+}\right) \subset B^{+}$and $\mathscr{M} \notin B^{+}$. This implies that $\pi_{i}^{-1}\left(T^{+}\right)$is $\left(2^{X}\right)^{+}$-closed. It now follows that $\pi_{i}$ is a cp mapping.

Let $h: \lambda X \rightarrow \mu X$ be the Jensen surjection.

ClaIm 7. For each $i \in N$ the mapping $h_{i}: \mu X \rightarrow \mu X_{i}$ defined by $h_{i}:=\varrho_{i} \circ \pi_{i} \circ h^{-1}$ is a well defined cp surjection.

Since the mappings $\pi_{i}$ and $\varrho_{i}$ are cp mappings (Claim 5 and Claim 6) this can be proved using the same technique as in the proof of Claim 5.

We now have the following diagram.

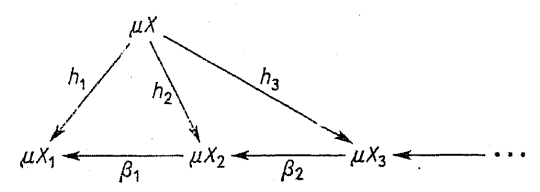

Since $\beta_{i} \circ h_{i+1}=\beta_{i} \circ \varrho_{i+1} \circ \pi_{i+1} \circ h^{-1}=\varrho_{i} \circ g_{i} \circ \varrho_{i+1}^{-1} \circ \varrho_{i+1} \circ \pi_{i+1} \circ h^{-1}$ $=\varrho_{i} \circ g_{i} \circ \pi_{i+1} \circ h^{-1}=\varrho_{i} \circ \pi_{i} \circ h^{-1}=h_{i}$ for each $i \in N$ the mapping $e: \mu X \rightarrow$ $\rightarrow \varliminf\left(\mu X_{i}, \beta_{i}\right)$ defined by $e(\mathscr{M})_{i}:=h_{i}(\mathscr{M})$ is a well defined continuous surjection. We claim that $e$ is one to one proving that $e$ is a homeomorphism. Indeed, take distinct $\mathscr{M}_{0}, \mathscr{M}_{1} \in \mu X$ and take disjoint $M_{0} \in \mathscr{M}_{0}$ and $M_{1} \in \mathscr{M}_{1}$. We may assume, without loss of generality that for each component $C$ of $X$ we have that $C$ is either contained in one of the $M_{i}$ 's or intersects both $T_{0}$ and $T_{1}$. As in the proof of Claim 5 one can derive that each component of $\lambda X$ is either contained in one of the $h^{-1}\left(M_{i}^{+}\right)$'s or intersects both $h^{-1}\left(M_{0}^{+}\right)$and $h^{-1}\left(M_{1}^{+}\right)$. There is an index $l \in N$ such that $\pi_{l} h^{-1}\left(M_{0}^{+}\right) \cap \pi_{l}^{-1} h^{-1}\left(M_{1}^{+}\right)=\varnothing$. Take two disjoint closed subsets $A_{0}, A_{1} \subset X_{l}$ such that $\pi_{l} h^{-1}\left(M_{i}^{+}\right) \subset A_{i}^{+}(i \in\{0,1\})$. First suppose that $A_{0}$ is clopen; then so is $A_{0}^{+}$ and consequently $\pi_{e} h^{-1}\left(M_{0}^{+}\right)=A_{0}^{+}$and $\pi_{l} h^{-1}\left(M_{1}^{+}\right)=\left(X_{l}-A_{0}\right)^{+}$(use the same technique as in the proof of Claim 5). In addition, it is easily seen that also $h^{-1}\left(M_{0}^{+}\right) \cup h^{-1}\left(M_{1}^{+}\right)=\lambda X$. Since $h$ extends the identity on $X$ we have that

$$
\begin{aligned}
\left(h^{-1}\left(M_{0}^{+}\right) \cap X\right) \cup\left(h^{-1}\left(M_{1}^{+}\right) \cap X\right) & =\left(M_{0}^{+} \cap X\right) \cup\left(M_{1}^{+} \cap X\right) \\
& =M_{0} \cup M_{1}=X
\end{aligned}
$$

which is a contradiction since $M_{0}$ and $M_{1}$ both belong to $\mathscr{T}(X)$. Therefore, we may assume that neither $A_{0}$ nor $A_{1}$ are clopen. Hence $A_{0}$ and $A_{1}$ both belong to $\mathscr{T}\left(X_{l}\right)$. It now follows that $\varrho_{l} \pi_{l} h^{-1}\left(M_{0}^{+}\right) \cap \varrho_{l} \pi_{l} h^{-1}\left(M_{1}^{+}\right)=\varnothing$. We conclude that $h_{l}\left(\mathscr{M}_{0}\right)$ $\neq h_{l}\left(\mathscr{M}_{1}\right)$.

Therefore $\mu X \approx \underline{\lim }\left(\mu X_{i}, \beta_{i}\right)$. By Theorem 5.2 the spaces $\mu X_{i}$ are Hilbert cubes. Since the mappings $\beta_{i}$ are cp mappings, they are cellular and applying Chapman's [4], [5] result again we conclude that $\mu X \approx Q$. 
6.5. COROLlary. Let $X$ be a nondegenerate compact connected polyhedron. Then $\lambda X$ is a Hilbert cube.

6.6. Corollary to Corollary. $\lambda X$ is a Hilbert cube if and only if $X$ is a nondegenerate metrizable continuum.

Proof. By a result in van Mill and Van de Vel [20], Corollary 6.5 suffices to prove 6.6.

We now prove the main result in this paper.

6.7. THEOREM. Let $X$ be a finite sum of nondegenerate metrizable continua. Then $\mu X$ is a Hilbert cube.

Proof. Let $C_{1}, \ldots, C_{n}$ be the collection of components of $X$. By Freudenthal's [11] expansion theorem, for each $i \leqslant n$ there is an inverse sequence

$$
P_{1}^{i} \stackrel{\beta_{1}^{i}}{\leftarrow} P_{2}^{i} \stackrel{\beta_{2}^{l}}{\leftarrow} P_{3}^{i} \stackrel{\beta_{3}^{l}}{\leftarrow} \ldots
$$

of compact connected nondegenerate polyhedra with onto bonding mappings $\beta_{j}^{i}$ $(j \in N)$ such that $\varliminf_{j}\left(P_{j}^{i}, \beta_{j}^{i}\right) \approx C_{i}$. For each $j \in N$ let $X_{j}$ be the disjoint topological sum of the $P_{j}^{i}$ s $(i \leqslant n)$. It is now straightforward to prove that

$$
X_{1} \stackrel{e_{1}}{\leftarrow} X_{2} \stackrel{e_{2}}{\leftarrow} X_{3} \stackrel{e_{3}}{\leftarrow} \ldots
$$

with bonding mappings defined in the obvious way, approximates $X$. This implies, by an obvious argument, that $\mu X \approx \varliminf\left(\mu X_{i}, \mu\left(\varrho_{i}\right)\right)$, where $\mu\left(\varrho_{i}\right)$ is the Jensen extension of $\varrho_{i}$. Using Proposition 6.3 and the cellularity of the $\mu\left(\varrho_{i}\right)$ 's yields $\mu X \approx \underline{\varliminf}\left(\mu X_{i}, \mu\left(\varrho_{i}\right)\right) \approx Q$.

For each $n \in N$ let $\lambda(n)$ be the cardinality of $\lambda\{1,2, \ldots, n\}$. Using the same technique as in Section 3 one can prove that if $X$ is a sum of $n$ copies of $[0,1]$ then $\lambda X$ is homeomorphic to a sum of $\lambda(n)$ copies of $Q$. Going through the whole process again, one then gets the following result.

6.8. THEOREM. Let $X$ be a sum of $n$ nondegenerate metrizable continua. Then $\lambda X$ is a sum of $\lambda(n)$ Hilbert cubes.

Details are left to the reader.

6.9. Corollary. Let $X$ be a compact metric space. The following statements are equivalent

(i) $\lambda X$ is a $Q$-manifold;

(ii) $X$ is a sum of finitely many nondegenerate continua.

The number $\lambda(n)$ is only known for $n \leqslant 7$ (cf. Verbeek [27]). It is an intriguing combinatorial problem to calculate the numbers $\lambda(n)$; the only information we can give is that

$$
{ }^{2} \log \lambda(n) \sim\left(\begin{array}{c}
n \\
{[n / 2]}
\end{array}\right) \frac{2^{n}}{\sqrt{2 \pi n}} .
$$

(cf. Verbeek [27]). Since $\lambda(3)=4$ and $\lambda(4)=12$ (cf. Verbeek [27]) there is no compact metric space $X$ for which $\lambda X$ is a sum of $i$ Hilbert cubes, where $4<i<12$.

Added in proof. The main result in this paper that $\lambda X \approx Q$ can also be derived by using a recent characterization of the Hilbert cube due to $H$. Torunczyk. This was observed independently by $\mathrm{C}$. Bruce Hughes and the author.

\section{References}

[0] R. H. Bing, Partioning a set, Bull. Amer. Math. Soc. 55 (1949), pp. 1101-1110.

[1] K. Borsuk, Theory of Shape, Warszawa 1975.

[2] M. Brown, Some applications of an approximation theorem for inverse limits, Proc. Amer. Math. Soc. 11 (1960), pp. 478-483.

[3] T. A. Chapman, On the structure of Hilbert cube manifolds, Comp. Math. 24 (1972), pp. 329-353.

[4] - Cell-like mappings of Hilbert cube manifolds: applications to simple homotopy type, Bull Amer. Math. Soc. 79 (1973), pp. 1286-1291.

[5] - Cell-like mappings of Hilbert cube manifolds: solution of a handle problem, Gen. Top. Appl. 5 (1975), pp. 123-145.

[6] - Lectures on Hilbert cube manifolds, Regional Conf. Ser. in Math., 28, Amer. Math. Soc., Providence, RI (1975).

[7] D. W. Curtis and R. M. Schori, $2^{X}$ and $C(X)$ are homeomorphic to the Hilbert cube, Bull. Amer. Math. Soc. 80 (1974), pp. 927-931.

[8] - - Hyperspaces which characterize simple homotopy type, Gen. Top. Appl. 6 (1976), pp. 153-165.

[9] - - Hyperspaces of polyhedra are Hilbert cubes (to appear).

[10] - - Hyperspaces of Peano continua are Hilbert cubes (to appear).

[11] H. Freudenthal, Entwicklungen von Räumen und ilren Gruppen, Comp. Math. 4 (1937), pp. $145-234$.

[12] O. H. Keller, Die Homoimorphie der kompakten konvexen mengen in Hilbertschen Raum, Math. Ann. 105 (1931), pp. 748-758.

[13] E. Michael, Topologies on spaces of subsets, Trans. Amer. Math. Soc. 71 (1951), pp. 152-182.

[14] J. van Mill, The superextension of the closed unit interval is homeomorphic to the Hilbert cube, Fund. Math. 103 (1979), pp. 151-175.

[15] - A pseudo-interior of $\lambda I$, Comp. Math. 36 (1978), pp. 75-82.

[16] - Supercompactness and Wallman spaces, Mathematical Centre Tract 85, Amsterdam 1977.

[17] - and M. Van de Vel, Subbases, convex sets and hyperspaces, (to appear in Pacific J. Math.).

[18] - - Contractibility, path connectedness and lc properties of superextensions, Bull. Acad. Polon. Sci. 26 (1978), pp. 261-269.

[19] - - Convexity preserving mappings in subbase convexity theory, Proc. Kon. Nad. Akad. Wet. A 81 (1978), pp. 76-90.

[20] - - On superextensions and hyperspaces, Top. Structures 2, Math Centre Tract. 115 (1979), pp. 169-180.

[21] J. Mioduszewski, Mappings of inverse limits, Colloq. Math. 10 (1963), pp. 39-44.

[22] R. M. Schori and J. E. West, $2^{I}$ is homeomorphic to the Hilbert cube, Bull. Amer. Math. Soc. 78 (1972), pp. $402-406$ 
[23] R. M. Schori and J. E. West, Hyperspaces of graphs are Hilbert cubes, Pacific J. Math. 53 (1974), pp. 239-251.

[24] - - The hyperspace of the closed unit interval is a Hilbert cube, Trans. Amer. Math. Soc. 213 (1975), pp. 217-235.

[25] A. Szymariski, Note on spaces with binary normal subbases (to appear).

[26] M. Van de Vel, Superextensions and Lefschetz fixed point structures, Fund. Math. 104 (1979), pp. 33-48.

[27] A. Verbeek, Superextensions of topological spaces, Mathematical Centre Tract. 41, Amsterdam 1972.

[28] M. Wojdysławski, Retractes absolus et hyperspaces des continus, Fund. Math. 32 (1939), pp. 184-192.

\section{DEPARTMENT OF MATHEMATICS}

UNIVERSITY OF WISCONSIN

\section{Madison, Wisconsin}

Current address:

SUBFACULTEIT WISKUNDE

VRIJE UNIVERSITEIT

De Boelelaan 1081

Amsterdam, The Netherlands

\section{Some combinatorial properties of ultrafilters}

by

\author{
Jussi Ketonen (Honolulu, Hawaii)
}

Abstract. Three unrelated combinatorial results are proved: (1) A result relating non-regular ultrafilters to weakly normal ultrafilters; (2) A partitioning property for indecomposable ultrafilters over singular cardinals and (3) A large cardinal-type result for inaccessible cardinals carrying indecomposable ultrafilters.

0. Introduction. Our notation and terminology follows that of the more recent set-theoretic literature. In particular $\alpha, \beta, \gamma, \ldots$ are variables for ordinals while $x, \lambda, \mu, \ldots$ are reserved for cardinals. The notation $|x|$ refers to the cardinality of the set $x$ and so on. An ultrafilter over a cardinal is always assumed to be uniform.

0.1 . Definition. An ultrafilter $D$ over $x$ is $(\lambda, \mu)$-regular if $\lambda \leqslant \mu$ and there is a set $S \subseteq D$ of power $\mu$ such that

$$
T \subseteq S \quad \text { and } \quad \lambda \leqslant|T| \rightarrow \cap T=0 .
$$

$D$ is $\mu$-regular if it is $(\omega, \mu)$-regular. $D$ is regular if it is $\varkappa$-regular.

This concept is due to Keisler. It measures the "width" of an ultrafilter. It is diametrically opposite to the notion of completeness of ultrafilters. It is a wellknown fact that the existence of suitably complete ultrafilters implies the existence of normal ultrafilters. In the case of simply non-regular ultrafilters we have to replace the condition of normality by a weaker one:

0.2 . Definition. An ultrafilter $D$ over $x$ is weakly normal if every pressing down function is bounded by a constant $<x$, i. e. if $f: x \rightarrow x$ s.t. $f<\operatorname{id}(\bmod D)$, then there is a $\xi<x$ s.t. $f \leqslant \xi(\bmod D)$.

Kanamori [3] was the first to show that suitably non-regular ultrafilters have weakly normal ultrafilters below them in the Rudin-Keisler order.

0.3. Definition. Given two ultrafilters $D, U$ over $\varkappa$ say $D \leqslant_{\mathrm{RK}} U$ if there is a function $f: x \rightarrow x$ s.t. $f_{*}(U)=D$; i.e. for all $x \subseteq x$ :

$$
x \in D \leftrightarrow f^{-1}(x) \in U
$$

Given $f, g: \varkappa \rightarrow \chi$ say $f \leqslant{ }_{\mathrm{RK}} g(\bmod D)$ if there is a function $\varphi: \varkappa \rightarrow \chi$ s.t. $f=\varphi \circ g$ 5 - Fundamenta Mathematicae T. CVII/3 